This item was submitted to Loughborough's Research Repository by the author.

Items in Figshare are protected by copyright, with all rights reserved, unless otherwise indicated.

\title{
Robust fault-tolerant tracking control for spacecraft proximity operations using time-varying sliding mode
}

PLEASE CITE THE PUBLISHED VERSION

https://doi.org/10.1109/TAES.2017.2729978

PUBLISHER

(C) IEEE

VERSION

AM (Accepted Manuscript)

LICENCE

CC BY-NC-ND 4.0

\section{REPOSITORY RECORD}

Hu, Qinglei, Xiaodong Shao, and Wen-Hua Chen. 2017. "Robust Fault-tolerant Tracking Control for Spacecraft Proximity Operations Using Time-varying Sliding Mode". figshare. https://hdl.handle.net/2134/26958. 


\title{
Robust Fault-tolerant Tracking Control for Spacecraft Proximity Operations Using Time-varying Sliding Mode
}

\author{
Qinglei Hu, Xiaodong Shao, Wen-hua Chen
}

\begin{abstract}
The capture of a tumbling free-floating object in orbit using an autonomous vehicle is a key technology for many future orbital missions. Spacecraft proximity operations will play an important role in the success of such missions. This paper technically presents a control approach for proximity operations between a target and a pursuer spacecraft that ensures accurate relative position tracking as well as attitude synchronization. Specifically, an integrated six degrees of freedom dynamics model is first established to describe the coupled relative motion of the pursuer with respect to the target. Then, a robust fault-tolerant tracking control scheme is proposed by combining the sliding mode control with the adaptive technique. It is proved that the control algorithm developed is not only robust against unexpected disturbances and adaptive to unknown and uncertain mass/inertia properties of the pursuer, but also able to accommodate a large class of actuator faults. In particular, by introducing a novel timevarying forcing function into the sliding dynamics, the designed controller is shown to guarantee the finite-time convergence of the translational and rotational tracking errors, and the convergence time as an explicit parameter can be assigned $a$ priori by the designer. Furthermore, a rigorous theoretical analysis is also presented in order to assess the fault-tolerance ability of the designed controller. Finally, numerous examples are carried out to evaluate the effectiveness and demonstrate the benefits of the overall control approach.
\end{abstract}

Index Terms -Finite-time convergence, time-varying sliding mode, fault-tolerant control, spacecraft proximity operations.

\section{INTRODUCTION}

$\mathrm{R}_{\mathrm{i}}$ ecent years have witnessed a tremendous research interest in the capture of a tumbling free-flying object in orbit using an autonomous vehicle, since this concept has been identified as an enabling technology for many near-future missions such as removing space debris, inspecting and repairing a malfunctioning satellite, refueling a powerless

This paragraph of the first footnote will contain the date on which you submitted your paper for review. It will also contain support information, including sponsor and financial support acknowledgment. For example, "This work was supported in part by the U.S. Department of Commerce under Grant BS123456".

Q. Hu and X. Shao are with the School of Automation Science and Electrical Engineering, Beihang University, Beijing, 100191, China (email: huql_buaa@buaa.edu.cn; xdshao_sasee@buaa.edu.cn)

W. Chen is with the Department of Aeronautical and Automotive Engineering, Loughborough University, LE11 3TU, UK (email: w.chen@lboro.ac.uk). satellite, and other space missions [1-3]. In 1998, the Engineering Test Satellite-VII (ETS-VII) successfully achieved the autonomous rendezvous and docking between two unmanned satellites under the funding and direction of Japan's National Space Development Agency, indicating that the autonomous technology is indeed feasible [4]. The use of autonomous system without ground or crew intervention would decrease the mission cost, and improve the mission frequency at the same time [5]; however, it poses great challenges for the guidance, navigation, and control subsystem onboard the maneuverable spacecraft. The latter primarily lies in the fact that accurate onboard sensing, dynamics models and control algorithms are needed for the rendezvous and docking mission. This paper seeks to present a control scheme for proximity operations between two spacecraft (namely a target and a pursuer) to provide a prerequisite for the successful capture of a tumbling target.

The research on spacecraft relative motion dynamics modeling begins with the linearized equations of relative translation proposed by Clohessy and Wiltshire for circular reference orbits [6]. Following the work of Clohessy and Wiltshire, many variants on the nonlinear models that are applicable to arbitrary orbital eccentricity were presented in [7]-[11] (to name just a few). However, most previous works have tended to focus on modeling the relative translation independently of relative rotational motion. In fact, for the proximity phase of the capture mission, the pursuer is required to track both time-varying relative position and reference attitude trajectories accurately and synchronously; on the other hand, it is widely-known that the coupling exists between the translational and rotational motions [12-15]; and as such it is favorable for proximity operations to describe the relative motion dynamics as the integrated six degrees-of-freedom (DOF) model. In recent years, the area of controller design for 6-DOF coupled relative motion of spacecraft has received a great deal of attention in the literature. Pan and Kapila [14] proposed a Lyapunov-based adaptive tracking control scheme for spacecraft formation flying. In their work, the mutual coupling in each spacecraft's translation and rotational motion induced by gravity torques was considered. Subbarao and Welsh [1] developed a nonlinear proportional-integralderivative (PID) type control law for motion synchronization of maneuverable spacecraft with respect to free-tumbling objects. Later, an extension of the work in [1] was presented in [15], 
wherein a robust sliding mode control strategy was synthesized and the dynamical coupling induced by the configuration of orbit-control thrusters was considered. A recent solution to the control problem of satellite proximity operations with a dual-quaternions-based description was founded in [16], where an adaptive tracking control strategy was derived. More recently, by combing the integral backstepping approach with the adaptive control technique, Sun and Huo [17] proposed a state feedback controller that guarantees the uniformly bounded convergence of the relative tracking errors. In [18], an adaptive integrated finite-time control scheme was presented for spacecraft translational and rotational motions subject to parametric uncertainties, external disturbances, and thruster misalignments. A similar result was also presented in [19]. The distinguishing feature residing in $[18,19]$ is that the controllers enable the spacecraft to track desired position and attitude in a pre-determined time. However, it is noteworthy that the benefit of this finite-time control architecture comes at a cost of heavy online computations.

Safety plays an important role in the success of autonomous rendezvous and docking missions. If anomalies, especially actuator faults, occur during the tracking process, it may result in aborting the mission or a series of potential problems such as excess fuel consumption, on-orbit collision [20], etc. Hence how to obtain a safe and reliable control in the event of actuator faults has been an active research topic that is of great practical significance. Related works on this problem include, but not limited to, [21]-[23] and the references therein. Cai et al. [21] proposed an indirect fault-tolerant control approach for spacecraft attitude tracking. By using the similar fault-tolerant control framework as that in [21], a finite-time fault-tolerant control scheme was introduced in [22] to achieve high precision attitude tracking of rigid spacecraft. A method for online generation of safe, fuel-optimized rendezvous trajectories was reported in [23], and the generated approach path guarantees collision avoidance for a large class of anomalous system behaviors. As far as we understand, however, there are a very limited number of available results were presented in the context of 6-DOF spacecraft relative motion. With regard to spacecraft autonomous rendezvous and docking, Jayaram [24] presented a robust fault-tolerant control strategy incorporating the fault detection, identification and recovery mechanism, but only the attitude actuator faults were considered in this work. Recently, a fault-tolerant control scheme with finite-time convergence was presented in [25] to address the spacecraft formation proximity operations.

To ensure a successful capture, accurate relative position tracking as well as attitude synchronization should be achieved within a finite time in the proximity phase of the mission, despite the presence of parametric uncertainties, external disturbances, and actuator faults. However, up to now, the controller design for spacecraft proximity operations with explicit consideration of these three issues and the finite-time convergence still remains open. In this paper, we present a robust finite-time fault-tolerant control scheme that provides a promising solution to this problem. The main contributions of this paper are summarized as follows:
1) The relative motion dynamics of spacecraft proximity operations is described as an Euler-Lagrange equation, in which the coupling effect between the translational and rotational motions is considered. The benefits of deriving this form of description are two-fold: first, the expression shares some well-known properties of Euler-Lagrange systems that can be exploited for control synthesis and stability analysis; secondly, it also helps to generalize the theoretical results of this work to a significant class of nonlinear systems whose dynamics can be expressed by Euler-Lagrange equations.

2) The control algorithm developed achieves accurate tracking control for spacecraft proximity operations in the event of parametric uncertainties, external disturbances and actuator faults. In particular, by introducing a novel time-varying forcing function into the sliding dynamics, the tracking errors are guaranteed to converge to zero within a prescribed time. The novelty of the forcing function mainly lies in its significantly different structure, which makes the sliding dynamics applicable to the faulty cases, and its design parameters less dependence on the initial condition.

3) A rigorous theoretical analysis is presented to evaluate the fault-tolerance ability of the proposed control scheme, where a key lemma is established that plays an important role in the analysis. The analysis results can provide a guideline for the designers to design a more comprehensive control strategy that ensures safety.

4) As opposed to most previous works, the proposed control scheme achieves robustness against external disturbances and parametric uncertainties with inexpensive online computations. This primarily benefits from the utilization of a norm-wise adaptive mechanism, in which only two parameters are required to be updated on-line.

The rest of this paper is organized as follows. Section II presents the problem description that involves the relevant coordinate frames, relative motion modeling, and control problem formulation. The controller design and the stability analysis, together with the analysis of the controller's fault-tolerance ability are given in Section III and followed by Section IV in which numerical simulations are performed to demonstrate the effectiveness of the proposed control scheme. Finally, we conclude this paper in Section V.

\section{Problem Description}

In this paper, we aim at providing an integrated control law for spacecraft proximity operations that ensures the success of the capture mission. Of the two involved spacecraft, termed as the target and the pursuer, only the pursuer is maneuverable. Although the target is uncontrollable, it is assumed that its state information including position, velocity, attitude and angular velocity is available to the pursuer in real time either estimated by the pursuer's onboard sensors or provided by the target or other sources. In the following subsection, the Cartesian coordinate frames, relative motion modeling, and control problem formulation are in order. Most definitions and descriptions are similar to the ones given in previous literature, they are still given here to make the paper self-contained. 

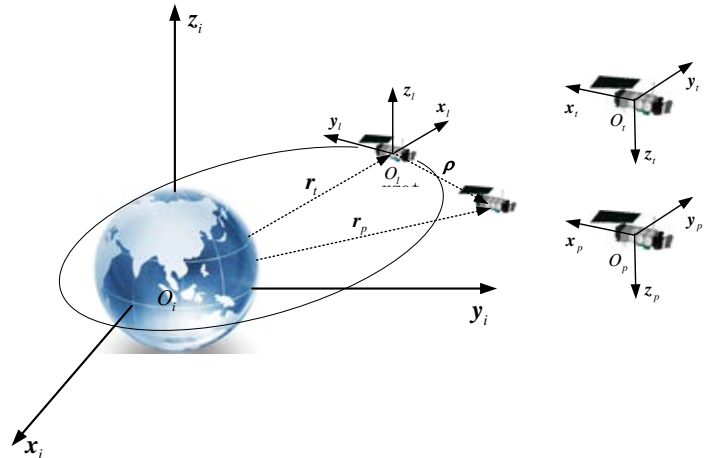

Fig. 1. Cartesian coordinate frames.

\section{A. Cartesian coordinate frames and notations}

To formulate the relative motion dynamics of the pursuer with respect to the target, four main coordinate frames are considered in this paper, as shown in Fig. 1.

1) Earth-centered inertial (ECI) frame: This frame is denoted as $\mathcal{F}_{i}=\left\{O_{i}, \boldsymbol{x}_{i}, \boldsymbol{y}_{i}, \mathbf{z}_{i}\right\}$, and its origin is located at the center of the Earth with $\boldsymbol{x}_{i}$ in the direction of the vernal equinox, $\boldsymbol{z}_{i}$ is directed along the celestial north pole, and $\boldsymbol{y}_{i}$ completes a right-handed orthogonal frame.

2) Local-vertical-local-horizontal (LVLH) frame: This frame defined as $\mathcal{F}_{l}=\left\{O_{l}, \boldsymbol{x}_{l}, \boldsymbol{y}_{l}, \mathbf{z}_{l}\right\}$ is attached to the mass center of the target spacecraft. $\boldsymbol{x}_{l}$ points radially outward from the Earth's center, $z_{l}$ is in the direction along the angular momentum of the orbit, and $\boldsymbol{y}_{l}=\mathbf{z}_{l} \times \boldsymbol{x}_{l}$ completes the right-handed reference frame.

3) Body-fixed frames: The frames $\mathcal{F}_{t}=\left\{O_{t}, \boldsymbol{x}_{t}, \boldsymbol{y}_{t}, \mathbf{z}_{t}\right\}$ and $\mathcal{F}_{p}=\left\{O_{p}, \boldsymbol{x}_{p}, \boldsymbol{y}_{p}, \mathbf{z}_{p}\right\}$ are defined for the target and the pursuer, respectively. The origin of each frame is located at the mass center of the corresponding spacecraft. For analysis purposes, without loss of generality, we assume that docking axis of the pursuer is in the direction along $\boldsymbol{x}_{p}$, and the outward normal at the receiving port on the target is aligned with $-\boldsymbol{x}_{t}$.

For notational compactness, the following notations are given.

1) $C_{a b}$ refers to the direction cosine matrix describing the rotation from the coordinate frame $\mathcal{F}_{b}$ to the frame $\mathcal{F}_{a}$, then a transformation of a vector from frame $\mathcal{F}_{b}$ to frame $\mathcal{F}_{a}$ can be conducted by $\boldsymbol{v}_{a}=\boldsymbol{C}_{a b} \boldsymbol{v}_{b}$.

2) The angular velocity vector of the frame $\mathcal{F}_{b}$ with respect to $\mathcal{F}_{a}$ is represented by $\omega_{a b}$, and its components expressed in either one of the coordinate frames are described as $\omega_{a b}^{a}$ or $\omega_{a b}^{b}$.

3) $\boldsymbol{I}_{3}$ is a $3 \times 3$ identity matrix, and $\|\not\|$ and $\|\not\|_{F}$ denote the standard Euclidean norm of a vector and the Frobenius norm of a matrix, respectively. The notation blkdiag $\{\boldsymbol{X}, \boldsymbol{Y}\}$ denotes a block matrix with the matrices $\boldsymbol{X}$ and $\boldsymbol{Y}$ on its main diagonal, whereas $(\square)^{\times} \in \square^{3}$ denotes the matrix representation of the linear cross-product operation such that $\boldsymbol{a}^{\times} \boldsymbol{b}=\boldsymbol{a} \times \boldsymbol{b}$ for any two vectors $\boldsymbol{a}, \boldsymbol{b} \in \square^{3}$.

\section{B. Relative translational dynamics}

The relative translational dynamics are derived based on the fundamental equations of the two-body problem. Let $\rho=\left[\rho_{x}, \rho_{y}, \rho_{z}\right]^{T}$ denote the relative position vector, then the relative translation can be governed by (cf. [10])

$$
\boldsymbol{M}_{t} \ddot{\boldsymbol{\rho}}+\boldsymbol{C}_{t}(\dot{v}) \dot{\boldsymbol{\rho}}+\boldsymbol{D}_{t}\left(\dot{v}, \ddot{v}, r_{p}\right) \boldsymbol{\rho}+\boldsymbol{n}_{t}\left(r_{t}, r_{p}\right)=\boldsymbol{f}_{t}+\boldsymbol{d}_{t}
$$

where $\boldsymbol{M}_{t}=m_{p} \boldsymbol{I}_{3}, \boldsymbol{C}_{t}(\dot{v})=m_{p} \boldsymbol{n}_{1}^{\times} \in S S$ (3) with $\boldsymbol{n}_{1}=[0,0,2 \dot{v}]^{T}$ is a Coriolis-like matrix, $\boldsymbol{D}_{t}\left(\dot{v}, \ddot{v}, r_{p}\right)=m_{p} \boldsymbol{a}_{1}\left(\dot{v}, \ddot{v}, r_{p}\right)$ can be viewed as a time-varying potential force, and $\boldsymbol{n}_{t}=m_{p} \boldsymbol{n}_{2}$ is a nonlinear term. The undefined terms appearing in the preceding descriptions are given by

$$
\boldsymbol{a}_{1}\left(\dot{v}, \ddot{v}, r_{p}\right)=\frac{\mu}{r_{p}^{3}} \boldsymbol{I}_{3}+\left[\begin{array}{ccc}
-\dot{v}^{2} & -\ddot{v} & 0 \\
\ddot{v} & -\dot{v}^{2} & 0 \\
0 & 0 & 0
\end{array}\right], \mathbf{n}_{2}=\mu\left[\frac{r_{t}}{r_{p}^{3}}-\frac{1}{r_{t}^{2}}, 0,0\right]^{T}
$$

In Eq. (1), $\boldsymbol{f}_{t}$ refers to the control force vector acting on the pursuer with its components expressed in the LVLH frame, and $\boldsymbol{d}_{t}$ is the disturbance force arising from $J_{2}$ perturbation, atmospheric drag, solar radiation pressure, thruster misalignment and so on. In addition, $m_{p}$ is the mass of the pursuer, $\mu$ is the gravitational constant of the Earth, $r_{t}$ denotes the distance between the mass center of the target and the Earth's center, and $r_{p}=\left[\left(r_{t}+\rho_{x}\right)^{2}+\rho_{y}^{2}+\rho_{z}^{2}\right]^{1 / 2}$ represents the distance from the centroid of the pursuer to the Earth's center. The evolutions of the true anomaly of the target and its rate are evaluated by

$$
\dot{v}=\frac{n(1+e \cos v(t))^{2}}{\left(1-e^{2}\right)^{3 / 2}}, \ddot{v}=\frac{-2 n^{2} e(1+e \cos v(t))^{3} \sin v(t)}{\left(1-e^{2}\right)^{3}}
$$

where $n=\sqrt{\mu / a^{3}}$ is the mean angular velocity of the target orbit, $a$ is the semimajor axis of the elliptical orbit, and $e$ is the orbital eccentricity.

\section{Relative rotational dynamics}

The governing equations for attitude motion of a freely tumbling target in terms of kinematics and dynamics are described by

$$
\dot{\boldsymbol{q}}_{t v}=\frac{1}{2}\left(\boldsymbol{q}_{t v}^{\times}+q_{t 4} \boldsymbol{I}_{3}\right) \boldsymbol{\omega}_{i t}^{t}, \dot{q}_{t 4}=-\frac{1}{2} \boldsymbol{q}_{t v}^{T} \boldsymbol{\omega}_{i t}^{t}
$$




$$
\boldsymbol{J}_{t} \dot{\boldsymbol{\omega}}_{i t}^{t}+\boldsymbol{\omega}_{i t}^{t} \times \boldsymbol{J}_{t} \boldsymbol{\omega}_{i t}^{t}=0
$$

where $\boldsymbol{q}_{t}:=\operatorname{col}\left(\boldsymbol{q}_{t v}, q_{t 4}\right) \in \square^{4}$ and $\boldsymbol{\omega}_{i t}^{t} \in \square^{3}$ denote the attitude quaternion and angular velocity of the target relative to the ECI frame respectively, and $\boldsymbol{J}_{t}$ is the inertia matrix defining the mass moment of inertia of the target.

Similarly, the governing equations for the pursuer's attitude dynamics can be expressed as (cf. [26]):

$$
\begin{gathered}
\dot{\boldsymbol{q}}_{p v}=\frac{1}{2}\left(\boldsymbol{q}_{p v}^{\times}+\boldsymbol{q}_{p 4} \boldsymbol{I}_{3}\right) \boldsymbol{\omega}_{i p}^{p}, \dot{q}_{p 4}=-\frac{1}{2} \boldsymbol{q}_{p v}^{T} \boldsymbol{\omega}_{i p}^{p} \\
\boldsymbol{J}_{p} \dot{\boldsymbol{\omega}}_{i p}^{p}=-\boldsymbol{\omega}_{i p}^{p} \times \boldsymbol{J}_{p} \boldsymbol{\omega}_{i p}^{p}+\boldsymbol{u}_{r}+\boldsymbol{d}_{r}
\end{gathered}
$$

where $\boldsymbol{q}_{p}:=\operatorname{col}\left(\boldsymbol{q}_{p v}, q_{p 4}\right) \in \square^{4}$ is the unit quaternion parameterzing the attitude orientation of the pursuer; $\boldsymbol{\omega}_{i p}^{p} \in \square^{3}$ is the angular velocity of the pursuer with respect to the ECI frame expressed in the pursuer's body-fixed frame; $\boldsymbol{J}_{p}$ is the mass moment of inertia of the pursuer; and $\boldsymbol{u}_{r}$ and $\boldsymbol{d}_{r}$ denote the control torque and the disturbance torque acting on the pursuer, respectively.

To address the attitude tracking issue for spacecraft proximity operations, we define $\boldsymbol{q}_{e}=\left[\boldsymbol{q}_{e v}^{T}, q_{e 4}\right]^{T} \in \square^{3} \times \square$ as the attitude tracking error that describes the relative orientation between the pursuer's body-fixed frame and the target's body-fixed frame, which is computed as [27]

$$
\boldsymbol{q}_{e}=\boldsymbol{q}_{p} \otimes \boldsymbol{q}_{t}^{*}=\left[\begin{array}{c}
q_{\mathrm{t} 4} \boldsymbol{q}_{p v}-q_{p 4} \boldsymbol{q}_{t v}+\boldsymbol{q}_{p v}^{\times} \boldsymbol{q}_{t v} \\
q_{t 4} q_{p 4}+\boldsymbol{q}_{t v}^{T} \boldsymbol{q}_{p v}
\end{array}\right]
$$

where $\boldsymbol{q}_{t}^{*}$ and $\otimes$ denote the conjugate quaternion of the unit quaternion $\boldsymbol{q}_{t}$ and the quaternion multiplication operator, respectively. The corresponding rotation matrix from the coordinate frame $\mathcal{F}_{t}$ to $\mathcal{F}_{p}$ is given by

$$
\boldsymbol{C}_{p t}=\left(q_{e 4}^{2}-\boldsymbol{q}_{e v}^{T} \boldsymbol{q}_{e v}\right) \boldsymbol{I}_{3}+2 \boldsymbol{q}_{e v} \boldsymbol{q}_{e v}^{T}-2 q_{e 4} \boldsymbol{q}_{e v}^{\times}
$$

Then, the relative angular velocity vector between the frames $\mathcal{F}_{p}$ and $\mathcal{F}_{t}$ is defined as

$$
\omega_{e}=\omega_{i p}^{p}-C_{p t} \omega_{i t}^{t}
$$

Hence, the tracking error dynamics for relative rotational motion can be derived that (cf. [21])

$$
\dot{\boldsymbol{q}}_{e v}=\frac{1}{2}\left(\boldsymbol{q}_{e v}^{\times}+q_{e 4} \boldsymbol{I}_{3}\right) \boldsymbol{\omega}_{e}, \dot{q}_{e 4}=-\frac{1}{2} \boldsymbol{q}_{e v}^{T} \boldsymbol{\omega}_{e}
$$

$$
\boldsymbol{J}_{p} \dot{\boldsymbol{\omega}}_{e}=-\left(\boldsymbol{\omega}_{i p}^{p}\right)^{\times} \boldsymbol{J}_{p} \boldsymbol{\omega}_{i p}^{p}+\boldsymbol{J}_{p}\left(\boldsymbol{\omega}_{e}^{\times} \boldsymbol{C}_{p t} \boldsymbol{\omega}_{i t}^{t}-\boldsymbol{C}_{p t} \dot{\boldsymbol{\omega}}_{i t}^{t}\right)+\boldsymbol{u}_{r}+\boldsymbol{d}_{r}
$$

Let $\boldsymbol{Q}=0.5\left(\boldsymbol{q}_{e v}^{\times}+q_{e 4} \boldsymbol{I}_{3}\right)$ and $\boldsymbol{P}=\boldsymbol{Q}^{-1}$. Then, after some algebraic manipulations, the above tracking error dynamics can be transformed to a more convenient representation with the form of (cf. [22])

$$
\boldsymbol{M}_{r} \ddot{\boldsymbol{q}}_{e v}+\boldsymbol{C}_{r} \dot{\boldsymbol{q}}_{e v}+\boldsymbol{P}^{T} \boldsymbol{H}_{2}=\boldsymbol{P}^{T}\left(\boldsymbol{u}_{r}+\boldsymbol{d}_{r}\right)
$$

where $\boldsymbol{M}_{r}=\boldsymbol{P}^{T} \boldsymbol{J}_{p} \boldsymbol{P}, \boldsymbol{C}_{r}=\boldsymbol{P}^{T} \boldsymbol{J}_{p} \dot{\boldsymbol{P}}-\boldsymbol{P}^{T}\left(\boldsymbol{J}_{p} \boldsymbol{P} \dot{\boldsymbol{q}}_{e v}\right)^{\times} \boldsymbol{P}, \quad \boldsymbol{H}_{2}=$ $\left(\boldsymbol{P} \dot{\boldsymbol{q}}_{e v}\right)^{\times} \boldsymbol{J}_{p}\left(\boldsymbol{C}_{p t} \boldsymbol{\omega}_{i t}^{t}\right)+\left(\boldsymbol{C}_{p t} \boldsymbol{\omega}_{i t}^{t}\right)^{\times} \boldsymbol{J}_{p}\left(\boldsymbol{P} \dot{q}_{e v}+\boldsymbol{C}_{p t} \boldsymbol{\omega}_{i t}^{t}\right)-\boldsymbol{J}_{p}\left(\boldsymbol{\omega}_{e}^{\times} \boldsymbol{C}_{p t} \boldsymbol{\omega}_{i t}^{t}\right.$ $\left.-C_{p t} \dot{\omega}_{i t}^{t}\right)$.

Remark 1: To ensure that $\boldsymbol{Q}$ defined in Eq. (13) is invertible so as to guarantee the validity of Eq. (13), the following condition must remain valid, $\forall t \geq 0$

$$
\operatorname{det}(2 \mathbf{Q})=q_{e 4}(t) \neq 0, \forall t \geq 0
$$

To this aim, we require that the initial condition be restricted such that $q_{e 4}(0) \neq 0$, and the subsequent control scheme be designed to guarantee $q_{e 4}(t) \neq 0$ holds for all time. As stated in [28], the restriction on the initial conditions is in fact very mild.

\section{6-DOF coupled relative motion dynamics}

For the proximity phase of the capture mission, accurate relative position and attitude tracking are required to align the relative position vector along the receiving port of the target while maintaining a constant relative distance between the target and the pursuer, and to reorient the pursuer in the desired orientation such that its docking port is always facing the receiving component of the target. To implement this, first, a virtual desired relative position vector $\boldsymbol{r}_{d}=\left[-r_{d}, 0,0\right]^{T}$ is introduced, and its components are expressed in the target's body-fixed frame. Then the translational position error can be defined as [1]

$$
\rho_{e}=\rho-\rho_{d}, \rho_{d}=C_{l t} \boldsymbol{r}_{d}
$$

where $\boldsymbol{C}_{l t}=\boldsymbol{C}_{l i} \boldsymbol{C}_{t i}^{T}$. Apparently, $\boldsymbol{\rho}_{d}$ is a time-varying vector and its direction in space depends directly upon the attitude orientation of the target. In addition, the orbit-control thrusters are fixed in the pursuer's body-fixed frame so that the control force vector in the LVLH frame is represented as

$$
\boldsymbol{f}_{t}=\boldsymbol{C}_{l p} \boldsymbol{u}_{t}
$$

where $\boldsymbol{u}_{t}$ denotes the control force vector expressed in the pursuer's body-fixed frame, and $\boldsymbol{C}_{l p}=\boldsymbol{C}_{l i} \boldsymbol{C}_{t i}^{T} \boldsymbol{C}_{p t}^{T}$ in which $\boldsymbol{C}_{t i}=\left(q_{t 4}^{2}-\boldsymbol{q}_{t v}^{T} \boldsymbol{q}_{t v}\right) \boldsymbol{I}_{3}+2 \boldsymbol{q}_{t v} \boldsymbol{q}_{t v}^{T}-2 q_{t 4} \boldsymbol{q}_{t v}^{\times}, \boldsymbol{C}_{p t}$ is given by Eq. (9), and 


$$
\begin{aligned}
\boldsymbol{C}_{l i}= & {\left[\begin{array}{ccc}
\cos (\omega+v) & \sin (\omega+v) & 0 \\
-\sin (\omega+v) & \cos (\omega+v) & 0 \\
0 & 0 & 1
\end{array}\right]\left[\begin{array}{ccc}
1 & 0 & 0 \\
0 & \cos i & \sin i \\
0 & -\sin i & \cos i
\end{array}\right] } \\
& \times\left[\begin{array}{ccc}
\cos \Omega & \sin \Omega & 0 \\
-\sin \Omega & \cos \Omega & 0 \\
0 & 0 & 1
\end{array}\right]
\end{aligned}
$$

where $\omega, \Omega$ and $i$ are the target orbital elements and denote the argument of perigee, the right ascension of ascending node and the inclination, respectively.

Then, the translational tracking error dynamics can now be rendered

$$
\boldsymbol{M}_{t} \ddot{\rho}_{e}+\boldsymbol{C}_{t}(\dot{v}) \dot{\boldsymbol{\rho}}_{e}+\boldsymbol{C}_{l p} \boldsymbol{H}_{1}=\boldsymbol{C}_{l p} \boldsymbol{u}_{t}+\boldsymbol{d}_{t}
$$

where $\quad \boldsymbol{H}_{1}=\boldsymbol{C}_{l p}^{T}\left(\boldsymbol{D}_{t}\left(\dot{v}, \ddot{v}, r_{p}\right) \boldsymbol{\rho}+\boldsymbol{n}_{t}\left(r_{t}, r_{p}\right)+\boldsymbol{M}_{t} \ddot{\boldsymbol{\rho}}_{d}+\boldsymbol{C}_{t}(\dot{v}) \dot{\boldsymbol{\rho}}_{d}\right)$, and the vectors $\dot{\rho}_{d}$ and $\ddot{\rho}_{d}$ appearing in $\boldsymbol{H}_{1}$ are given by [15],

$$
\dot{\rho}_{d}=C_{l t}\left(\boldsymbol{\omega}_{l t}^{t} \times \boldsymbol{r}_{d}\right), \ddot{\rho}_{d}=C_{l t}\left(\dot{\boldsymbol{\omega}}_{l t}^{t} \times \boldsymbol{r}_{d}+\boldsymbol{\omega}_{l t}^{t} \times \boldsymbol{\omega}_{l t}^{t} \times \boldsymbol{r}_{d}\right)
$$

where $\boldsymbol{\omega}_{l t}^{t}=\boldsymbol{\omega}_{i t}^{t}-\boldsymbol{C}_{l t}^{T} \boldsymbol{\omega}_{i l}^{l} \quad$ and $\quad \dot{\boldsymbol{\omega}}_{l t}^{t}=\dot{\boldsymbol{\omega}}_{i t}^{t}-\boldsymbol{C}_{l t}^{T} \dot{\boldsymbol{\omega}}_{i l}^{l}+\boldsymbol{\omega}_{l t}^{t}\left(\boldsymbol{C}_{l t}^{T} \boldsymbol{\omega}_{i l}^{l}\right)$ with $\boldsymbol{\omega}_{i l}^{l}=[0,0, \dot{\boldsymbol{v}}]^{T}$ and $\dot{\boldsymbol{\omega}}_{i l}^{l}=[0,0, \ddot{\boldsymbol{v}}]^{T}$.

To construct the integrated dynamics model for proximity operations, we define the state vector $\boldsymbol{e}=\left[\boldsymbol{\rho}_{e}^{T}, \boldsymbol{q}_{e v}^{T}\right]^{T} \in \square^{6}$. Then, by combing the translational tracking error dynamics in Eq. (18) with the relative rotational dynamics in Eq. (13), the 6-DOF relative motion dynamics is described as an Euler-Lagrange equation of the form

$$
M \ddot{e}+C \dot{e}+G=B(u+d)
$$

where $\boldsymbol{M}=\operatorname{blkdiag}\left\{\boldsymbol{M}_{t}, \boldsymbol{M}_{r}\right\}, \quad \boldsymbol{C}=\operatorname{blkdiag}\left\{\boldsymbol{C}_{t}, \boldsymbol{C}_{r}\right\}, \quad \boldsymbol{G}=$ $\left[\left(\boldsymbol{C}_{l p} \boldsymbol{H}_{1}\right)^{T},\left(\boldsymbol{P}^{T} \boldsymbol{H}_{2}\right)^{T}\right]^{T}, \boldsymbol{B}=\operatorname{blkdiag}\left\{\boldsymbol{C}_{l p}, \boldsymbol{P}^{T}\right\}, \quad \boldsymbol{u}=\left[\boldsymbol{u}_{t}^{T}, \boldsymbol{u}_{r}^{T}\right]^{T}$ and $\boldsymbol{d}=\left[\left(\boldsymbol{C}_{l p}^{T} \boldsymbol{d}_{t}\right)^{T}, \boldsymbol{d}_{r}^{T}\right]^{T}$.

From a practical viewpoint, the actuators mounted on the pursuer are always subject to actuator faults, and each of them might fail in the following four fault modes: Partial loss of energy (F1): Actuator can only respond partially to the expected control command; Continuous float (F2): Actuator cannot output the expected actuating power accurately, and there exists a small DC offset; Complete loss of energy (F3): Actuator completely fails to work; Locking (F4): Actuator has a fixed and uncontrolled output. With explicit consideration of the actuator faults F1-F4, the control input can then be mathematically modeled as

$$
\boldsymbol{u}=\boldsymbol{D}\left(\boldsymbol{E} \boldsymbol{u}_{c}+\boldsymbol{F}\right)
$$

where $\quad \boldsymbol{D}=\operatorname{blkdiag}\left\{\boldsymbol{D}_{1}, \boldsymbol{D}_{2}\right\} \in \square^{6 \times n} \quad$ denotes the control allocation matrix with $\boldsymbol{D}_{1} \in \square^{3 \times n_{1}}$ and $\boldsymbol{D}_{2} \in \square^{3 \times n_{2}}, n_{1}$ and $n_{2}$ are the numbers of the orbital actuators and attitude actuators, respectively; $\quad \boldsymbol{E}=\operatorname{blkdiag}\left\{\boldsymbol{E}_{1}, \boldsymbol{E}_{2}\right\} \in \square^{n \times n}$ is the actuation effectiveness matrix characterizing the health condition of the actuators with $\boldsymbol{E}_{i}=\operatorname{diag}\left(\delta_{i 1}, \delta_{i 2}, \ldots, \delta_{i n_{i}}\right) \in \square^{n_{i} \times n_{i}}, i=1,2$, each element of which satisfies $0 \leq \delta_{i j} \leq 1 ; \boldsymbol{F} \in \square^{6 \times n}$ represents the float or locking values of the actuators; and $\boldsymbol{u}_{c}$ is the control signal commanded by the control law to be designed later.

The dynamics model in Eq. (20) has the following useful properties [19]:

Property 1: The matrix $(\dot{\boldsymbol{M}}-2 \boldsymbol{C})$ is skew-symmetric, that is $\boldsymbol{x}^{T}(\dot{\boldsymbol{M}}-2 \boldsymbol{C}) \boldsymbol{x}=0$ for any vector $\boldsymbol{x} \in \square^{6}$.

Property 2: The inertial-like matrix $\boldsymbol{M}$ is symmetric and positive definite. Moreover, according to the theorem of Rayleigh-Ritz, it is straightforward to show that for all $\boldsymbol{x} \in \square^{6}$

$$
\lambda_{\text {min }}(\boldsymbol{M})\|\boldsymbol{x}\|^{2} \leq \boldsymbol{x}^{T} \boldsymbol{M} \boldsymbol{x} \leq \lambda_{\text {max }}(\boldsymbol{M})\|\boldsymbol{x}\|^{2}
$$

where $\lambda_{\min }(\boldsymbol{M})$ and $\lambda_{\max }(\boldsymbol{M})$ are the minimum and maximum eigenvalues of $\boldsymbol{M}$, respectively.

Remark 2: An implicit assumption in Property 1 is that the time derivative of the mass of the pursuer is close to zero. The fully details regarding the assumption will appear hereafter (see Assumption 1).

Remark 3: In fact, the relative translational motion is affected by the rotational one due to the dependence of the thrust force vector for translational motion control on the pursuer's attitude and the thruster configuration, as witnessed by Eqs. (16)-(21). It is just the dynamic coupling that this paper is mainly concerned with. Therefore, it can be claimed that the formula (20) is a 6-DOF coupled dynamics equation in which the coupling effect between the translational and rotational motions is included.

Remark 4: The relative motion dynamics of spacecraft proximity operations is formulated as a 6-DOF Lagrange-like model as given by Eq. (20), in which the coupling effect between the translational and rotational motions is explicitly considered. In particular, the derived dynamics model with the typical form of the Euler-Lagrange equation has two main advantages. First, this expression has some well-known properties of the Euler-Lagrange systems as summarized in Properties 1-2 that will be employed in control synthesis and stability analysis (see Sec. III.B for more details). Secondly, it can be utilized to describe a wide range of dynamic systems in practice, such as spacecraft system, robotic system, etc. As such, the theoretical results of this work can be readily extended to more general systems whose dynamics can be expressed as Euler-Lagrange equations.

Assumption 1: Assume that the mass $m_{p}$ and mass moment of inertia $\boldsymbol{J}_{p}$ of the pursuer are constant (or slowly varying over time), but otherwise unknown due to fuel consumption and payload variations.

Assumption 2: The composite disturbance $\boldsymbol{d}$ and the additive actuator fault $\boldsymbol{F}$ are assumed to be unknown but bounded, and satisfy $\|\boldsymbol{D F}+\boldsymbol{d}\| \leq d_{m}$, where $d_{m}$ is a positive constant. 
Remark 5: Assumption 1 primarily indicates that the changes of the mass and inertial of the pursuer are very slow for close proximity operations, which is reasonable in practice. In addition, it should be noted that the additive actuator faults are bounded due to the physical structure features of the actuators, and thus Assumption 2 is reasonable as well.

\section{E. Control objective}

The control objective is to develop a robust fault-tolerant tracking control scheme for spacecraft proximity operations to nullify the translational and rotational tracking errors in a finite time $T$, i.e., $\lim _{t \rightarrow T} \boldsymbol{e}=\mathbf{0}$ and $\lim _{t \rightarrow T} \dot{\boldsymbol{e}}=\mathbf{0}$, in the presence of external disturbances, parametric uncertainties and possible actuator faults.

\section{CONTROL LAW DESIGN AND STABILITY ANALYSIS}

A. A novel time-varying sliding mode manifold: design and convergence analysis

By following the approach outlined in [29], a novel time-varying sliding mode manifold is defined here as follows

$$
\boldsymbol{s}=\dot{\boldsymbol{e}}(t)+k \boldsymbol{e}(t)-\boldsymbol{f}(t)
$$

where $k$ is a positive scalar, and $\boldsymbol{f}(t)=\left[f_{1}(t), f_{2}(t), \ldots, f_{6}(t)\right]^{T}$ is referred to as the forcing function in sliding dynamics with $f_{i}(t), i=1,2, \ldots, 6$ given by

$$
\begin{aligned}
& f_{i}(t) \\
& =\left\{\begin{array}{lr}
\dot{e}_{i}(0)+k e_{i}(0), & 0 \leq t \leq t_{k} \\
\left(\dot{e}_{i}(0)+k e_{i}(0)\right)\left[1-\tau_{i}\left(1-\cos \left(\frac{\pi\left(t-t_{k}\right)}{\left(t_{m}-t_{k}\right)}\right)\right)\right], & t_{k}<t \leq t_{m} \\
\frac{1}{2}\left(\dot{e}_{i}(0)+k e_{i}(0)\right)\left(1-2 \tau_{i}\right)\left(1+\cos \left(\frac{\pi\left(t-t_{m}\right)}{\left(t_{f}-t_{m}\right)}\right)\right), & t_{m}<t \leq t_{f} \\
0, & t>t_{f}
\end{array}\right.
\end{aligned}
$$

where $\dot{e}_{i}(0)$ and $e_{i}(0)$ refer to the initial values of $\dot{e}_{i}(t)$ and $e_{i}(t)$ respectively, $t_{f}$ is the terminal time specified $a$ priori by the designer according to mission requirement, $t_{k}$ and $t_{m}$ are the constant time parameters with $t_{k}<t_{m}<t_{f}$, and $\tau_{i}$ is a constant parameter to be determined (see Eq. (29) shown later). The forcing function $f(t)$ has the following three properties:

P1) $\boldsymbol{f}(0)=\boldsymbol{f}\left(t_{k}\right)=\dot{\boldsymbol{e}}(0)+k \boldsymbol{e}(0)$. With the satisfaction of this condition, it is not difficult to check that the system states originate from the sliding regime, i.e., $\boldsymbol{s}(0)=\mathbf{0}$, and that $\dot{\boldsymbol{e}}\left(t_{k}\right)+k \boldsymbol{e}\left(t_{k}\right)=\boldsymbol{f}\left(t_{k}\right)=\dot{\boldsymbol{e}}(0)+k \boldsymbol{e}(0)$ if an appropriate control law is derived that ensures the state trajectories return to the sliding regime within $t_{k}$ and stays on it thereafter. The latter is useful in conducting the finite-time convergence analysis, which will become clear later.
P2) For $t \geq t_{f}, \boldsymbol{f}(t) \equiv \mathbf{0}$. This implies that the asymptotic stability of the closed-loop system.

P3) The forcing function is continuous and differentiable throughout the entire response although it is piecewise defined, and its first time derivative is bounded. This property is one of the basic requirements for the existence of a sliding control.

Lemma 1: Consider the time-varying sliding dynamics defined by Eq. (22). If an appropriate control law is derived that ensures the state trajectories reach the sliding regime within $t_{k}$ and stay on it thereafter, then the relative errors will converge to zero at the terminal time $t_{f}$, that is, $\boldsymbol{e} \equiv \mathbf{0}$ and $\dot{\boldsymbol{e}} \equiv \mathbf{0}$ for all $t \geq t_{f}$

Proof: If the system states reach the sliding regime within $t_{k}$, and remain on it thereafter, i.e., $\boldsymbol{s}(t) \equiv \mathbf{0}$ for all $t \geq t_{k}$, then we have

$$
\dot{e}_{i}(t)+k e_{i}(t)=f_{i}(t), i=1,2, \ldots, 6 \forall t \geq t_{k}
$$

It is apparent from Eqs. (23) and (24) that $\dot{e}_{i}\left(t_{k}\right)+k e_{i}\left(t_{k}\right)=$ $\dot{e}_{i}(0)+k e_{i}(0)$. Note that the forcing function $f_{i}(t)$ is a piecewise function, and thus the subsequent error response has to be analyzed separately in three different periods corresponding to the three time intervals over which $f_{i}(t)$ has been described in detail by Eq. (23).

For $t_{k}<t \leq t_{m}$, solving Eq. (24) with $\lim _{t \rightarrow t_{k}^{+}} e_{i}(t)=e_{i}(0)$ yields

$$
\begin{aligned}
e_{i}(t)= & \frac{p_{1 i}\left(1-\tau_{i}\right)}{k}+\left(e_{i}(0)-\frac{p_{1 i}\left(1-\tau_{i}\right)}{k}-\frac{p_{1 i} \tau_{i} k}{p_{2}}\right) e^{-k\left(t-t_{k}\right)} \\
& +\frac{p_{1 i} \tau_{i} \pi}{p_{2}\left(t_{m}-t_{k}\right)} \sin \left(\pi \frac{\left(t-t_{k}\right)}{\left(t_{m}-t_{k}\right)}\right)+\frac{p_{1 i} \tau_{i} k}{p_{2}} \cos \left(\pi \frac{\left(t-t_{k}\right)}{\left(t_{m}-t_{k}\right)}\right)
\end{aligned}
$$

where $p_{1 i}=\dot{e}_{i}(0)+k e_{i}(0)$ and $p_{2}=k^{2}+\left(\pi /\left(t_{m}-t_{k}\right)\right)^{2}$.

In view of Eq. (25), the value of $e_{i}(t)$ at $t=t_{m}$ can then be obtained by direct calculation

$$
\begin{aligned}
e_{i}\left(t_{m}\right) & =\frac{p_{1 i}\left(1-\tau_{i}\right)}{k} \\
+ & \left(e_{i}(0)-\frac{p_{1 i}\left(1-\tau_{i}\right)}{k}-\frac{p_{1 i} \tau_{i} k}{p_{2}}\right) e^{-k\left(t_{m}-t_{k}\right)}-\frac{p_{1 i} \tau_{i} k}{p_{2}}
\end{aligned}
$$

Regarding the time interval $t_{m}<t \leq t_{f}$, solving Eq. (24) and using the preceding, we have

$$
\begin{aligned}
e_{i}(t)= & \frac{p_{1 i}\left(1-2 \tau_{i}\right)}{2 k}+\left[e_{i}\left(t_{m}\right)-\frac{p_{1 i}\left(1-2 \tau_{i}\right)}{2 k}-\frac{p_{1 i}\left(1-2 \tau_{i}\right) k}{2 p_{3}}\right] \\
& +\frac{p_{1 i} \pi\left(1-2 \tau_{i}\right)}{2 p_{3}\left(t_{f}-t_{m}\right)} \sin \left(\pi \frac{\left(t-t_{m}\right)}{\left(t_{f}-t_{m}\right)}\right)+\frac{p_{1 i}\left(1-2 \tau_{i}\right) k}{2 p_{3}} \cos \left(\pi \frac{\left(t-t_{m}\right)}{\left(t_{f}-t_{m}\right)}\right)
\end{aligned}
$$


where $p_{3}=k^{2}+\left(\pi /\left(t_{f}-t_{m}\right)\right)^{2}$.

Substituting $t=t_{f}$ into Eq. (27), we can get

$$
\begin{aligned}
e_{i}\left(t_{f}\right)= & {\left[\frac{p_{1 i}\left(1-\tau_{i}\right)}{k}-\frac{p_{1 i} \tau_{i} k}{p_{2}}-\frac{p_{1 i}\left(1-2 \tau_{i}\right)}{2 k}-\frac{p_{1 i}\left(1-2 \tau_{i}\right) k}{2 p_{3}}\right] } \\
& \times e^{-k\left(t_{f}-t_{m}\right)}+\left[e_{i}(0)-\frac{p_{1 i}\left(1-\tau_{i}\right)}{k}-\frac{p_{1 i} \tau_{i} k}{p_{2}}\right] e^{-k\left(t_{f}-t_{k}\right)} \\
& +\frac{p_{1 i}\left(1-2 \tau_{i}\right)}{2 k}-\frac{p_{1 i}\left(1-2 \tau_{i}\right) k}{2 p_{3}}
\end{aligned}
$$

Let us further take $e_{i}\left(t_{f}\right)=0$, then the constant $\tau_{i}$ residing in the forcing function can be deduced as

$$
\tau_{i}=\frac{\left(\frac{e_{i}(0)}{p_{1 i}}-\frac{1}{k}\right) e^{-k\left(t_{f}-t_{k}\right)}+\left(\frac{1}{2 k}-\frac{k}{2 p_{3}}\right) e^{-k\left(t_{f}-t_{m}\right)}+\frac{1}{2 k}-\frac{k}{2 p_{3}}}{\left(\frac{k}{p_{2}}-\frac{1}{k}\right) e^{-k\left(t_{f}-t_{k}\right)}+\left(\frac{1}{p_{2}}-\frac{1}{p_{3}}\right) k e^{-k\left(t_{f}-t_{m}\right)}+\frac{1}{k}-\frac{k}{p_{3}}}
$$

Obviously, with the choice of the constant parameters $\tau_{i}$, $i=1,2, \ldots, 6$ described by Eq. (29), the sliding dynamics leads to $\boldsymbol{e}\left(t_{f}\right)=\mathbf{0}$. As an immediate result, it can be trivially shown that $\dot{\boldsymbol{e}}\left(t_{f}\right)=\mathbf{0}$ due to $\dot{\boldsymbol{e}}\left(t_{f}\right)+\boldsymbol{k} \boldsymbol{e}\left(t_{f}\right)=\boldsymbol{f}\left(t_{f}\right)=\mathbf{0}$. Further, by solving Eq. (24) with $\lim _{t \rightarrow t_{f}^{+}} \boldsymbol{e}(t)=\mathbf{0}$, we can obtain $\boldsymbol{e}(t)=\mathbf{0}$ and $\dot{\boldsymbol{e}}(t)=\mathbf{0}$ for all $t>t_{f}$. Based on the above argument, we can finally conclude the finite-time convergence of the relative errors, i.e., $\boldsymbol{e} \equiv \mathbf{0}$ and $\dot{\boldsymbol{e}} \equiv \mathbf{0}$ for all $t \geq t_{f}$. This completes the proof.

Looking at the expression in Eq. (29), the value of $\tau_{i}$ can be determined by assigning two positive values satisfying $t_{k}<t_{m}<$ $t_{f}$ to $t_{k}$ and $t_{m}$. It is notable that $t_{k}$ has to be chosen large enough to ensure the system states reach the sliding regime within $t_{k}$ under an appropriate controller. Generally speaking, there is no unique choice for $t_{m}$, and decreasing $t_{m}$ renders a faster convergence rate but an increased control effort during the time interval $t_{k}<t \leq t_{m}$ and vice versa. Note however that typical actuators used for spacecraft control are always subject to magnitude constraints, thus one should select appropriate values for $t_{k}$ and $t_{m}$ so as to ensure that the control input signals will not exceed such constraints.

In the following, we will discuss the time responses of the sliding motion with various choices of the time parameters $t_{m}$ and $t_{f}$ under the assumption that $t_{k}=0$. For example, similar to [29], given $k=10, e_{i}(0)=-1$ and $\dot{e}_{i}(0)=0$, the error responses with the novel time-varying sliding dynamics in terms of various values of $t_{m}$ and $t_{f}$ are shown in Fig. 2, and the corresponding forcing functions are shown in Fig. 3. It is seen that the tracking error converges invariably to zero at the specified terminal time $t_{f}$, although different $t_{m}$ are chosen.
Additionally, it can also be seen that decreasing the value of $t_{m}$ contributes to a faster convergence rate during the initial period.
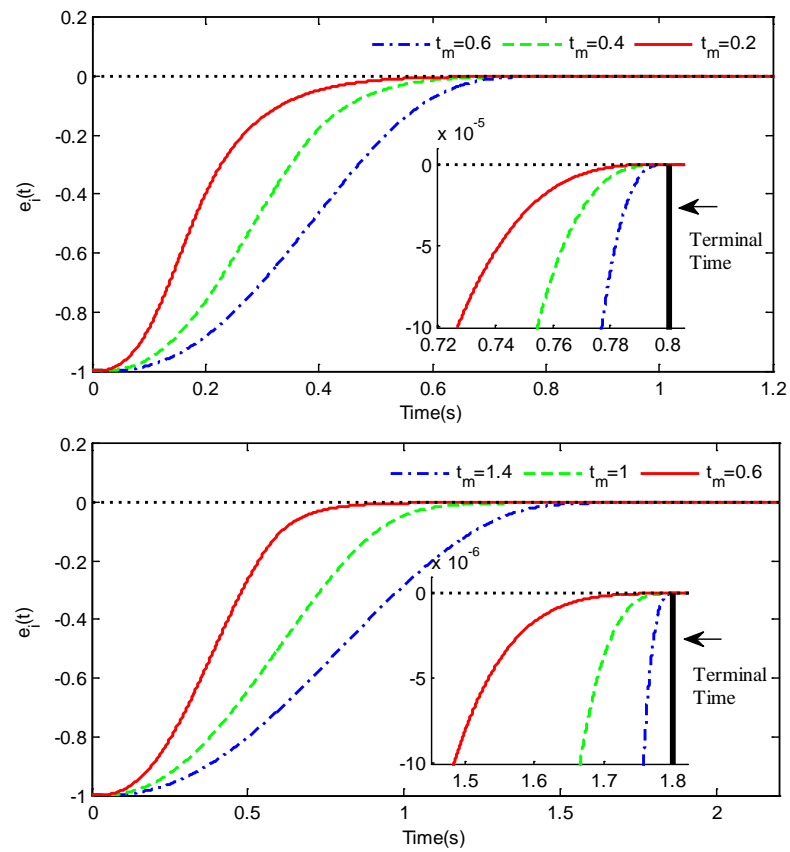

Fig. 2. Time responses of the sliding motion with different $t_{m}$ (top subplot: $t_{f}$ $=0.8 \mathrm{~s}$; bottom subplot: $t_{f}=1.8 \mathrm{~s}$ ).
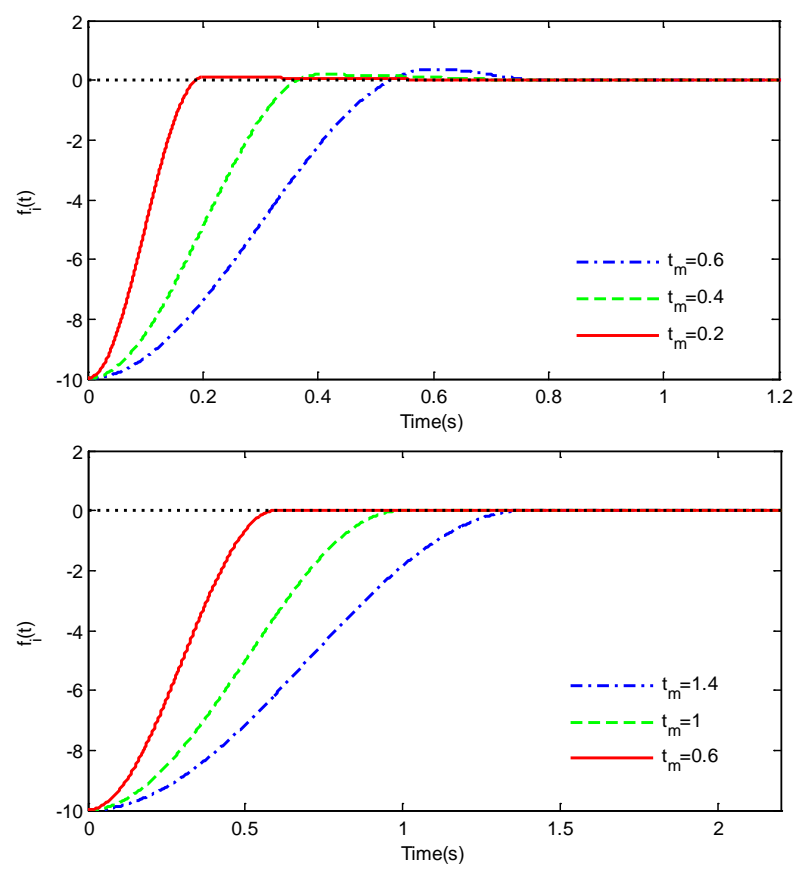

Fig. 3. The forcing functions with different $t_{m}$ (top subplot: $t_{f}=0.8 \mathrm{~s}$; bottom subplot: $\left.t_{f}=1.8 \mathrm{~s}\right)$.

Remark 6: From a practical viewpoint, guaranteeing the tracking errors converge to zero within a pre-specified time is a practical design aspect that is involved less in previous researches but is nonetheless crucial to the successful achievement of spacecraft proximity operations. Inspired by [29], we artfully introduce a novel time-varying forcing function into the sliding dynamics to guarantee that the 
translational and rotational tracking errors converge to zero within a fixed finite time, which can be assigned $a$ priori by the designer according to mission requirement. The novelty of the forcing function (23), compared to the one used in [29], mainly lies in two aspects: a) it has a significantly different structure such that the sliding dynamics developed in this paper can be used to the case in which actuator faults are considered; b) the parameters $\tau_{i}, i=1,2, \ldots, 6$ have less dependence on the initial condition (see Eq. (29)).

\section{B. Tracking controller design and stability analysis}

To facilitate the control law derivation, an auxiliary error vector is defined as

$$
\boldsymbol{e}_{r}=\left[\boldsymbol{e}_{r 1}^{T}, \boldsymbol{e}_{r 2}^{T}\right]^{T}=\boldsymbol{s}-\dot{\boldsymbol{e}}(t)
$$

where $\boldsymbol{e}_{r 1} \in \square^{3}$ and $\boldsymbol{e}_{r 2} \in \square^{3}$.

Taking the time derivative of $\boldsymbol{s}$ defined by Eq. (22) along the relative motion dynamics (20), and using the definition of $\boldsymbol{e}_{r}$, the open-loop system can be rewritten as

$$
M \dot{s}+C s=B(u+d)+M \dot{e}_{r}+C e_{r}-\boldsymbol{G}
$$

For the sake of simplicity, the term $\left(\boldsymbol{M} \dot{\boldsymbol{e}}_{r}+\boldsymbol{C} \boldsymbol{e}_{r}-\boldsymbol{G}\right)$ on the right-hand side of Eq. (31) is denoted as $\boldsymbol{R}=\left[\boldsymbol{R}_{1}^{T}, \boldsymbol{R}_{2}^{T}\right]^{T}$, with $\boldsymbol{R}_{1} \square \boldsymbol{M}_{t} \dot{\boldsymbol{e}}_{r 1}+\boldsymbol{C}_{t} \boldsymbol{e}_{r 1}-\boldsymbol{C}_{l p} \boldsymbol{H}_{1}$ and $\boldsymbol{R}_{2} \square \boldsymbol{M}_{r} \dot{\boldsymbol{e}}_{r 2}+\boldsymbol{C}_{r} \boldsymbol{e}_{r 2}-\boldsymbol{P}^{T} \boldsymbol{H}_{2}$. It is evident from the structure of the matrices $\boldsymbol{M}, \boldsymbol{C}$ and $\boldsymbol{G}$ that a linear parameterization of the unknown parameters $m_{p}$ as well as $\boldsymbol{J}_{p}$ for $\boldsymbol{R}$ is possible. To this end, the linear parameterizations are performed separately for $\boldsymbol{R}_{1}$ and $\boldsymbol{R}_{2}$, and the separate derivations are merely to facilitate the illustration.

With regard to $\boldsymbol{R}_{1}$, it is a straightforward matter to have

$$
\boldsymbol{R}_{1} \square \boldsymbol{M}_{t} \dot{\boldsymbol{e}}_{r 1}+\boldsymbol{C}_{t} \boldsymbol{e}_{r 1}-\boldsymbol{C}_{l p} \boldsymbol{H}_{1}=\boldsymbol{C}_{l p} \boldsymbol{Y}_{t} m_{p}
$$

where $\boldsymbol{Y}_{t}=\boldsymbol{C}_{l p}^{T}\left[\dot{\boldsymbol{e}}_{r 1}+\boldsymbol{n}_{1}^{\times} \boldsymbol{e}_{r 1}-\left(\boldsymbol{a}_{1} \boldsymbol{\rho}+\boldsymbol{n}_{2}+\ddot{\boldsymbol{\rho}}_{d}+\boldsymbol{n}_{1}^{\times} \dot{\boldsymbol{\rho}}_{d}\right)\right]$. To linearly parameterize $\boldsymbol{R}_{2}$, a linear operator $\boldsymbol{L}(\square): \square^{3} \rightarrow \square^{3 \times 6}$ is first introduced, and for any $\boldsymbol{x}=\left[x_{1}, x_{2}, x_{3}\right]^{T}$, there always has $\boldsymbol{J}_{p} \boldsymbol{x}=\boldsymbol{L}(\boldsymbol{x}) \boldsymbol{\vartheta}$, where $\boldsymbol{\vartheta}=\left[J_{11}^{p}, J_{12}^{p}, J_{13}^{p}, J_{22}^{p}, J_{23}^{p}, J_{33}^{p}\right]^{T}$ with $J_{i j}^{p}$ being the elements of $\boldsymbol{J}_{p}$. Based on this manipulation, $\boldsymbol{R}_{2}$ can then be linearly parameterized as

$$
\boldsymbol{R}_{2} \square \boldsymbol{M}_{r} \dot{\boldsymbol{e}}_{r 2}+\boldsymbol{C}_{r} \boldsymbol{e}_{r 2}-\boldsymbol{P}^{T} \boldsymbol{H}_{2}=\boldsymbol{P}^{T} \boldsymbol{Y}_{r} \boldsymbol{\vartheta}
$$

where $\boldsymbol{Y}_{r}$ is a known regression matrix that is described by $\boldsymbol{Y}_{r}=\boldsymbol{L}\left(\boldsymbol{P} \dot{\boldsymbol{e}}_{r 2}\right)+\boldsymbol{L}\left(\dot{\boldsymbol{P}}_{r 2}\right)+\left(\boldsymbol{P} \boldsymbol{e}_{r 2}\right)^{\times} \boldsymbol{L}\left(\omega_{e}\right)-\left(\boldsymbol{C}_{p t} \omega_{i t}^{t}\right)^{\times} \boldsymbol{L}\left(\boldsymbol{\omega}_{e}+\boldsymbol{C}_{p t} \boldsymbol{\omega}_{i t}^{t}\right)$ $-\boldsymbol{\omega}_{e}^{\times} \boldsymbol{L}\left(\boldsymbol{C}_{p t} \boldsymbol{\omega}_{i t}^{t}\right)+\boldsymbol{L}\left(\boldsymbol{\omega}_{e}^{\times} \boldsymbol{C}_{p t} \boldsymbol{\omega}_{i t}^{t}-\boldsymbol{C}_{p t} \dot{\boldsymbol{\omega}}_{i t}^{t}\right) \quad$ with $\dot{\boldsymbol{P}}$ given by $\dot{\boldsymbol{P}}=-\boldsymbol{P} \dot{\boldsymbol{Q}} \boldsymbol{P}$.

In view of Eqs. (32) and (33), it follows that

$$
\boldsymbol{R} \square \boldsymbol{M} \dot{\boldsymbol{e}}_{r}+\boldsymbol{C} \boldsymbol{e}_{r}-\boldsymbol{G}=\boldsymbol{B Y} \boldsymbol{\theta}
$$

where $\boldsymbol{Y}=\operatorname{blkdiag}\left\{\boldsymbol{Y}_{t}, \boldsymbol{Y}_{r}\right\}$ and $\boldsymbol{\theta}=\left[m_{p}, \boldsymbol{\vartheta}^{T}\right]^{T}$. According to Assumption 1 , it is clear that $\dot{\boldsymbol{\theta}}=\mathbf{0}$, which implies that the adaptive control technique can be employed to synthesize an online estimate of the unknown vector $\boldsymbol{\theta}$. However, there are seven unknown variables to be estimated when traditional adaptive technique is employed. This will inevitably result in expensive online computations. To remedy this, a norm-wise adaptive algorithm is derived for control design.

Theorem 1: Consider the coupled 6-DOF relative motion dynamics described by Eqs. (20) and (21) under Assumptions $1-2$. Assume that the actuators are suitably mounted along the pursuer's body-fixed frame, and that the matrix $\boldsymbol{D E D}^{T}$ remains positive definite for all fault scenarios under consideration. The control law and adaptive law are synthesized as

$$
\begin{gathered}
\boldsymbol{u}_{c}=\boldsymbol{D}^{T}\left(-k_{1}\|\boldsymbol{s}\| \frac{\boldsymbol{B}^{T} \boldsymbol{s}}{\left\|\boldsymbol{B}^{T} \boldsymbol{s}\right\|^{2}}-\hat{\boldsymbol{b}}^{T} \boldsymbol{\Phi} \frac{\boldsymbol{B}^{T} \boldsymbol{s}}{\left\|\boldsymbol{B}^{T} \boldsymbol{s}\right\|}\right) \\
\dot{\hat{\boldsymbol{b}}}=\eta\left(\boldsymbol{\Phi}\left\|\boldsymbol{B}^{T} \boldsymbol{s}\right\|-\boldsymbol{\kappa} \hat{\boldsymbol{b}}\right)
\end{gathered}
$$

where $s$ denotes the sliding dynamics defined by Eq. (22), $k_{1}$, $\eta$ and $\kappa$ are positive design parameters, $\hat{\boldsymbol{b}}$ is the estimation of $\boldsymbol{b}$ which is defined as $\boldsymbol{b}=\left[\|\boldsymbol{\theta}\|, d_{m}\right]^{T}$, and $\boldsymbol{\Phi}=\left[\|\boldsymbol{Y}\|_{F}, 1\right]^{T}$. If $k_{1}, \eta$ and $\kappa$ are appropriately chosen such that the system states reach the sliding regime within $t_{k}$, then based on Lemma 1 the finite-time convergence of the relative errors is achieved, that is, $\boldsymbol{e} \equiv \mathbf{0}$ for all time $t \geq t_{f}$.

Proof: Consider the following Lyapunov function candidate

$$
V=\frac{1}{2} \boldsymbol{s}^{T} \boldsymbol{M} \boldsymbol{s}+\frac{1}{2 \eta \sigma}(\boldsymbol{b}-\sigma \hat{\boldsymbol{b}})^{T}(\boldsymbol{b}-\sigma \hat{\boldsymbol{b}})+\frac{\boldsymbol{\kappa}^{-1} \beta^{2}}{8 \eta \sigma} \boldsymbol{b}^{T} \boldsymbol{b}
$$

where $\sigma=\lambda_{\min }\left(\boldsymbol{D E} \boldsymbol{D}^{T}\right)$ is the minimum eigenvalue of DED $\boldsymbol{D}^{T}$.

Taking the time derivative of $V$ leads to

$$
V=\frac{1}{2} \boldsymbol{s}^{T} \boldsymbol{M} \boldsymbol{s}+\frac{1}{2 \eta \sigma}(\boldsymbol{b}-\sigma \hat{\boldsymbol{b}})^{T}(\boldsymbol{b}-\sigma \hat{\boldsymbol{b}})
$$

where $\sigma=\lambda_{\min }\left(\boldsymbol{D} \boldsymbol{E} \boldsymbol{D}^{T}\right)$ is the minimum eigenvalue of DED $\boldsymbol{D}^{T}$.

Taking the time derivative of $V$ leads to

$$
\dot{V}=\boldsymbol{s}^{T} \boldsymbol{M} \dot{\boldsymbol{s}}+\frac{1}{2} \boldsymbol{s}^{T} \dot{\boldsymbol{M}} \boldsymbol{s}-\frac{1}{\eta}(\boldsymbol{b}-\sigma \hat{\boldsymbol{b}})^{T} \dot{\hat{\boldsymbol{b}}}
$$


In terms of Eq. (31), it follows that

$\dot{V}=\boldsymbol{s}^{T} \boldsymbol{B}\left(\mathrm{DE} \boldsymbol{u}_{c}+\mathrm{DF}+\boldsymbol{d}\right)-\boldsymbol{s}^{T} \boldsymbol{C} \boldsymbol{s}+\frac{1}{2} \boldsymbol{s}^{T} \dot{\boldsymbol{M}} \boldsymbol{s}+\boldsymbol{s}^{T} \boldsymbol{R}-\frac{1}{\eta}(\boldsymbol{b}-\sigma \hat{\boldsymbol{b}})^{T} \dot{\hat{\boldsymbol{b}}}$

According to Property 1, it is straightforward to obtain

$$
\boldsymbol{s}^{T}(\dot{M}-2 \boldsymbol{C}) \boldsymbol{s}=0
$$

Then, according to Assumption 2 and using the above relationship, Eq. (39) reduces to

$$
\begin{aligned}
\dot{V} & =\boldsymbol{s}^{T} \boldsymbol{B D E} \boldsymbol{u}_{c}+\boldsymbol{s}^{T} \boldsymbol{B}(\boldsymbol{D F}+\boldsymbol{d})+\boldsymbol{s}^{T} \boldsymbol{B Y} \boldsymbol{\theta}-\frac{1}{\eta}(\boldsymbol{b}-\sigma \hat{\boldsymbol{b}})^{T} \dot{\hat{\boldsymbol{b}}} \\
& \leq \boldsymbol{s}^{T} \boldsymbol{B D E} \boldsymbol{u}_{c}+\left\|\boldsymbol{B}^{T} \boldsymbol{s}\right\| d_{m}+\left\|\boldsymbol{B}^{T} \boldsymbol{s}\right\|\|\boldsymbol{Y} \boldsymbol{\theta}\|-\frac{1}{\eta}(\boldsymbol{b}-\sigma \hat{\boldsymbol{b}})^{T} \dot{\hat{\boldsymbol{b}}}
\end{aligned}
$$

Owing to the fact that the vector norm $\|[||$ is compatible with the induced matrix norm $\|\not\|_{F}$, it follows that $\|\boldsymbol{Y} \boldsymbol{\theta}\| \leq\|\boldsymbol{Y}\|_{F}\|\boldsymbol{\theta}\|$. Thus Eq. (41) can be further expressed as

$$
\dot{V} \leq \boldsymbol{s}^{T} \boldsymbol{B D E} \boldsymbol{u}_{c}+\left\|\boldsymbol{B}^{T} \boldsymbol{s}\right\| \boldsymbol{b}^{T} \boldsymbol{\Phi}-\frac{1}{\eta}(\boldsymbol{b}-\sigma \hat{\boldsymbol{b}})^{T} \dot{\hat{\boldsymbol{b}}}
$$

Inserting the control law (35) and the adaptive law (36) into Eq. (42) and simplifying the resulting equation yield

$$
\dot{V} \leq-k_{1} \sigma\|\boldsymbol{s}\|+\kappa(\boldsymbol{b}-\sigma \hat{\boldsymbol{b}})^{T} \hat{\boldsymbol{b}}
$$

By resorting to the properties of square sum, we have the following inequality for any positive scalar $l>0.5$ :

$$
\begin{aligned}
\kappa(\boldsymbol{b}-\sigma \hat{\boldsymbol{b}})^{T} \hat{\boldsymbol{b}} & =-\frac{\kappa}{\sigma}(\boldsymbol{b}-\sigma \hat{\boldsymbol{b}})^{T}(\boldsymbol{b}-\sigma \hat{\boldsymbol{b}}-\boldsymbol{b}) \\
& \leq-\frac{\kappa(2 l-1)}{2 \sigma l}(\boldsymbol{b}-\sigma \hat{\boldsymbol{b}})^{T}(\boldsymbol{b}-\sigma \hat{\boldsymbol{b}})+\frac{\kappa l}{2 \sigma} \boldsymbol{b}^{T} \boldsymbol{b}
\end{aligned}
$$

Then, with the consideration of the above inequality and by virtue of Property 2, Eq. (43) becomes

$$
\begin{gathered}
\dot{V} \leq-k_{1} \sigma\|\boldsymbol{s}\|-\frac{\kappa(2 l-1)}{2 \sigma l}(\boldsymbol{b}-\sigma \hat{\boldsymbol{b}})^{T}(\boldsymbol{b}-\sigma \hat{\boldsymbol{b}})+\frac{\kappa l}{2 \sigma} \boldsymbol{b}^{T} \boldsymbol{b} \\
\leq-c V^{\frac{1}{2}}+\frac{\kappa(2 l-1)}{2 \sigma l}\left[\left((\boldsymbol{b}-\sigma \hat{\boldsymbol{b}})^{T}(\boldsymbol{b}-\sigma \hat{\boldsymbol{b}})\right)^{1 / 2}\right. \\
\left.-(\boldsymbol{b}-\sigma \hat{\boldsymbol{b}})^{T}(\boldsymbol{b}-\sigma \hat{\boldsymbol{b}})\right]+\frac{\kappa l}{2 \sigma} \boldsymbol{b}^{T} \boldsymbol{b}
\end{gathered}
$$

where $c:=\min \left\{k_{1} \sigma\left(2 / \lambda_{\max }(\boldsymbol{M})\right)^{1 / 2},(\kappa(2 l-1) / 2 \sigma l) \sqrt{2 \eta \sigma}\right\}$.

For further analysis, the following two cases are considered. Case A: If $(\boldsymbol{b}-\sigma \hat{\boldsymbol{b}})^{T}(\boldsymbol{b}-\sigma \hat{\boldsymbol{b}}) \geq 1$, then we have

$$
\left((\boldsymbol{b}-\sigma \hat{\boldsymbol{b}})^{T}(\boldsymbol{b}-\sigma \hat{\boldsymbol{b}})\right)^{1 / 2}-(\boldsymbol{b}-\sigma \hat{\boldsymbol{b}})^{T}(\boldsymbol{b}-\sigma \hat{\boldsymbol{b}}) \leq 0
$$

Case B: If $(\boldsymbol{b}-\sigma \hat{\boldsymbol{b}})^{T}(\boldsymbol{b}-\sigma \hat{\boldsymbol{b}})<1$, then by completing the square we obtain

$$
\left((\boldsymbol{b}-\sigma \hat{\boldsymbol{b}})^{T}(\boldsymbol{b}-\sigma \hat{\boldsymbol{b}})\right)^{1 / 2}-(\boldsymbol{b}-\sigma \hat{\boldsymbol{b}})^{T}(\boldsymbol{b}-\sigma \hat{\boldsymbol{b}}) \leq 0.25
$$

Based on the above arguments, it can be concluded from Eq. (45) that

$$
\dot{V} \leq-c V^{1 / 2}+\beta
$$

where $\beta=(\kappa(2 l-1) / 8 \sigma l)+(\kappa l / 2 \sigma) \boldsymbol{b}^{T} \boldsymbol{b}$.

Note that Eq. (48) can be further changed into the form $\dot{V} \leq-V^{1 / 2}\left(c-\beta V^{-1 / 2}\right)$, which indicates that if $c-\beta V^{-1 / 2}>0$, the region $V \leq(\beta / c)^{2}$ can be reached in finite time. On the other hand, it is evident that $V \leq(\beta / c)^{2}$ is in fact an invariable set, since $\dot{V} \leq 0$ on the level set $V=(\beta / c)^{2}$. Therefore, no matter whether $V(0) \leq(\beta / c)^{2}$ or not, we can claim that $V$ and hence $(\boldsymbol{b}-2 \sigma \hat{\boldsymbol{b}})$ are bounded for all $t \geq 0$. Then, owing to the boundedness of $(\boldsymbol{b}-2 \sigma \hat{\boldsymbol{b}})$ and $\boldsymbol{b}$, it is not difficult to verify that $\hat{\boldsymbol{b}}$ is bounded. In what follows, we will explore fully the finite-time stability of the closed- loop system. To this aim, we shall introduce a key lemma that is useful for subsequent analysis.

Lemma 2 [34]: Consider a system $\dot{\boldsymbol{x}}=f(\boldsymbol{x}, \boldsymbol{u})$ and a Lyapunov function candidate $V_{s}(\boldsymbol{x})$. Suppose that there exist scalars $a>0,0<\gamma<1$ and $0<\phi<\infty$ such that

$$
\dot{V}_{s} \leq-a V_{s}^{\gamma}(x)+\phi
$$

Then, the system state can converge to the residual set given by

$$
V_{s} \leq(\phi / a(1-\theta))^{\frac{1}{\gamma}}, \forall t \geq T
$$

where $T \leq V_{s}\left(x_{0}\right)^{1-\gamma} /(a \theta(1-\gamma))$ is a finite time, $V_{s}\left(x_{0}\right)$ is the initial value of $V_{s}(x)$, and $0<\theta<1$.

Then, according to Lemma 2, we can conclude from Eq. (48) that

$$
V(t) \leq\left(\beta / c\left(1-\theta_{0}\right)\right)^{2}, \forall t \geq t_{T}
$$

where $t_{T}=2 V(0)^{1 / 2} /\left(c \theta_{0}\right), V(0)$ is the initial value of $V$, and $0<\theta_{0}<1$. It is noteworthy that the size of the residual set $V(t) \leq\left(\beta / c\left(1-\theta_{0}\right)\right)^{2}$ can be made arbitrarily small by appropriately choosing $k_{1}, \eta$ and $\kappa$, so that we have $\boldsymbol{s}(t) \square \mathbf{0}$ 
for all $t \geq t_{T}$, which implies that the sliding condition holds. Then, based on Lemma 1, we finally draw the conclusion that the relative errors can converge to zero within the specified terminal time $t_{f}$, i.e., $\lim _{t \rightarrow t_{f}} \boldsymbol{e}=\mathbf{0}, \lim _{t \rightarrow t_{f}} \dot{\boldsymbol{e}}=\mathbf{0}$. Henceforth, the proof of Theorem 1 has been completed.

Remark 7: In practice, an infinite switching frequency cannot be obtained due to limited actuator bandwidth, thus the proposed control scheme suffers from the chattering problem. Furthermore, careful inspection of Eq. (35) shows that a potential singularity exists in the designed controller. Hence, to attenuate the chattering phenomenon and overcome the singularity problem, by following the approach outlined in [30], the control law (35) is modified as

$$
\boldsymbol{u}_{c}=\boldsymbol{D}^{T}\left(-k_{1}\|\boldsymbol{s}\| \frac{\boldsymbol{B}^{T} \boldsymbol{s}}{\left\|\boldsymbol{B}^{T} \boldsymbol{s}\right\|^{2}+\varepsilon}-\hat{\boldsymbol{b}}^{T} \boldsymbol{\Phi} \frac{\boldsymbol{B}^{T} \boldsymbol{s}}{\left\|\boldsymbol{B}^{T} \boldsymbol{s}\right\|+\varepsilon}\right)
$$

where $\varepsilon$ is a small positive scalar.

Remark 8: A systematic procedure for selecting the design parameters is summarized below.

Step 1: Select a positive scalar $k$ for the time-varying sliding dynamics.

Step 2: Assign $t_{f}$ according to mission requirement, and choose time parameters $t_{k}$ and $t_{m}$ in accordance with $t_{k}<t_{m}<t_{f}$. As discussed in Sec. III.A, a compromise between convergence rate and magnitude constraints should be reached by appropriate choice of $t_{k}$ and $t_{m}$, which is of practical engineering significance.

Step 3: Choose sufficient large $k_{1}$ and $\eta$ for the control law (52) and adaptive law (36), respectively, such that $t_{k} \geq t_{T}=2 V(\mathbf{0})^{1 / 2} /\left(c \theta_{0}\right)$ is strictly guaranteed. One caveat here is that although the residual set of $V$ can be made arbitrarily small by choosing proper control parameters, it requires the increase of $k_{1}$ and $\eta$, together with the extension of $t_{T}$. Thus, designer should face a tradeoff between control accuracy and energy efficiency, and meanwhile choose a sufficient large $t_{k}$ in Step 2 so as to ensure the condition $t_{k} \geq t_{T}$ holds.

Step 4: Choose a small positive $\varepsilon$. Note that if a smaller $\varepsilon$ is selected for a higher accuracy, more serious chattering will be resulted. Thus, one should balance the control performance and chattering problem through a suitable choice of $\varepsilon$. Besides, the designer should take a small and nonzero $\kappa$ in order to ensure the boundedness of $\hat{\boldsymbol{b}}$.

\section{Analysis of the controller's fault-tolerance ability}

As stated in Theorem 1, the ability of the proposed control scheme to account for actuator faults requires that $\boldsymbol{D E} \boldsymbol{D}^{T}$ remains positive definite (i.e. $\sigma=\lambda_{\text {min }}\left(\boldsymbol{D E} \boldsymbol{D}^{T}\right)>0$ ) for all fault scenarios under consideration. This naturally leads to the question of which fault cases can make this requirement hold true. At first glance, it looks like a trivial problem since some discussions have been made in the literature, such as $[21,22]$.
However, in that respect, most of the conclusions are empirical and not all-inclusive due to the lack of rigorous theoretical support. In this subsection, we will answer this question from a mathematical viewpoint.

To proceed the subsequent analysis, we establish a key Lemma as follows.

Lemma 3: Suppose that $\boldsymbol{D}$ is of full-row rank (by proper placement of the actuators on the pursuer). Then, for the given fault cases, $\boldsymbol{D} \boldsymbol{E} \boldsymbol{D}^{T}$ remains positive definite if and only if the following relationship holds true

$$
\operatorname{rank}(\boldsymbol{D E})=6
$$

Proof: The detailed proof is presented in Appendix.

It follows from Lemma 3 that the sufficient and necessary condition for $\sigma>0$ is

$$
\operatorname{rank}(\boldsymbol{D E})=6
$$

From Eq. (21), we have $\boldsymbol{D E}=\operatorname{blkdiag}\left\{\boldsymbol{D}_{1} \boldsymbol{E}_{1}, \boldsymbol{D}_{2} \boldsymbol{E}_{2}\right\}$. Then, it is clear that the condition described by Eq. (53) is equivalent to the fact that $\operatorname{rank}\left(\boldsymbol{D}_{1} \boldsymbol{E}_{1}\right)=3$ and $\operatorname{rank}\left(\boldsymbol{D}_{2} \boldsymbol{E}_{2}\right)=3$ hold simultaneously. Since the distribution matrices $\boldsymbol{D}_{i}, i=1,2$ can be made full-row rank by proper placement of the actuators on the pursuer, $\operatorname{rank}\left(\boldsymbol{D}_{i}\right)=3$ can be readily guaranteed. Additionally, note that given $\boldsymbol{D}_{i}$ and $\boldsymbol{E}_{i}$, there always has $\operatorname{rank}\left(\boldsymbol{D}_{i} \boldsymbol{E}_{i}\right) \leq \min \left\{\operatorname{rank}\left(\boldsymbol{D}_{i}\right), \operatorname{rank}\left(\boldsymbol{E}_{i}\right)\right\}$. Thus, to guarantee the condition (53), the following two conditions must be simultaneously guaranteed

$$
\operatorname{rank}\left(\boldsymbol{E}_{1}\right) \geq 3, \operatorname{rank}\left(\boldsymbol{E}_{2}\right) \geq 3
$$

which means that the numbers of orbit actuators and attitude actuators suffering from F3 or F4 are no more than $n_{1}-3$ and $n_{2}-3$, respectively. However, care must be taken that the conditions as in Eq. (54) is a necessary but not sufficient condition for $\operatorname{rank}(\boldsymbol{D E})=6$; in other words, $\operatorname{rank}\left(\boldsymbol{D}_{i} \boldsymbol{E}_{i}\right)=3$ may not hold even if $\operatorname{rank}\left(\boldsymbol{E}_{i}\right) \geq 3$ is satisfied, this will be verified by the detailed examples shown later.

Based on the above arguments, we finally conclude that the proposed control scheme can accommodate such fault cases in which at most $n_{1}-3$ orbit actuators and $n_{2}-3$ attitude actuators suffer from F3 or F4, and $\operatorname{rank}(\boldsymbol{D E})=6$ holds. Of course, the crucial premise of this is that the remaining active actuators are still able to provide a sufficient actuating power for the pursuer to perform given manipulations. For the other cases in which the condition $\sigma>0$ is not satisfied, the system becomes under-actuated; this situation is out of the main scope of this paper.

The following numerical examples are presented to illustrate the fault-tolerance ability of the proposed controller. For illustration, we take only the attitude control subsystem as 
example, and assume that the attitude maneuver of the pursuer is actuated by six thrusters distributed on the pursuer in such a way [21]

$$
\boldsymbol{D}_{2}=\left[\begin{array}{cccccc}
0.8 & -0.8 & 0 & 0 & 0 & 0 \\
0 & 0 & 0.7 & -0.7 & 0 & 0 \\
0 & 0 & 0 & 0 & 0.7 & -0.7
\end{array}\right]
$$

and that the maximum available thrust force of each thruster is $1 \mathrm{~N}$. Then, given four actuation effectiveness matrices $\boldsymbol{E}_{2}^{1}=\operatorname{diag}([0.5,0.7,0.7,0.2,0.3,0.5]), \boldsymbol{E}_{2}^{2}=\operatorname{diag}([0,0.5,0.7,0$, $0.7,0]), \quad \boldsymbol{E}_{2}^{3}=\operatorname{diag}([0,0.5,0.7,0,0,0])$ and $\boldsymbol{E}_{2}^{4}=\operatorname{diag}([0,0$, $0.5,0.7,0.7,0]$ ), and the superscript $i$ is used here to denote different fault cases. The ranks of $\boldsymbol{D}_{2}$ and $\boldsymbol{D}_{2} \boldsymbol{E}_{2}$ under different fault cases specified by the four effectiveness matrices are given in Table I, and the corresponding attainable sets of control torques are apparent by inspecting the 3-D geometric figures shown in Fig. 4.

TABLE I

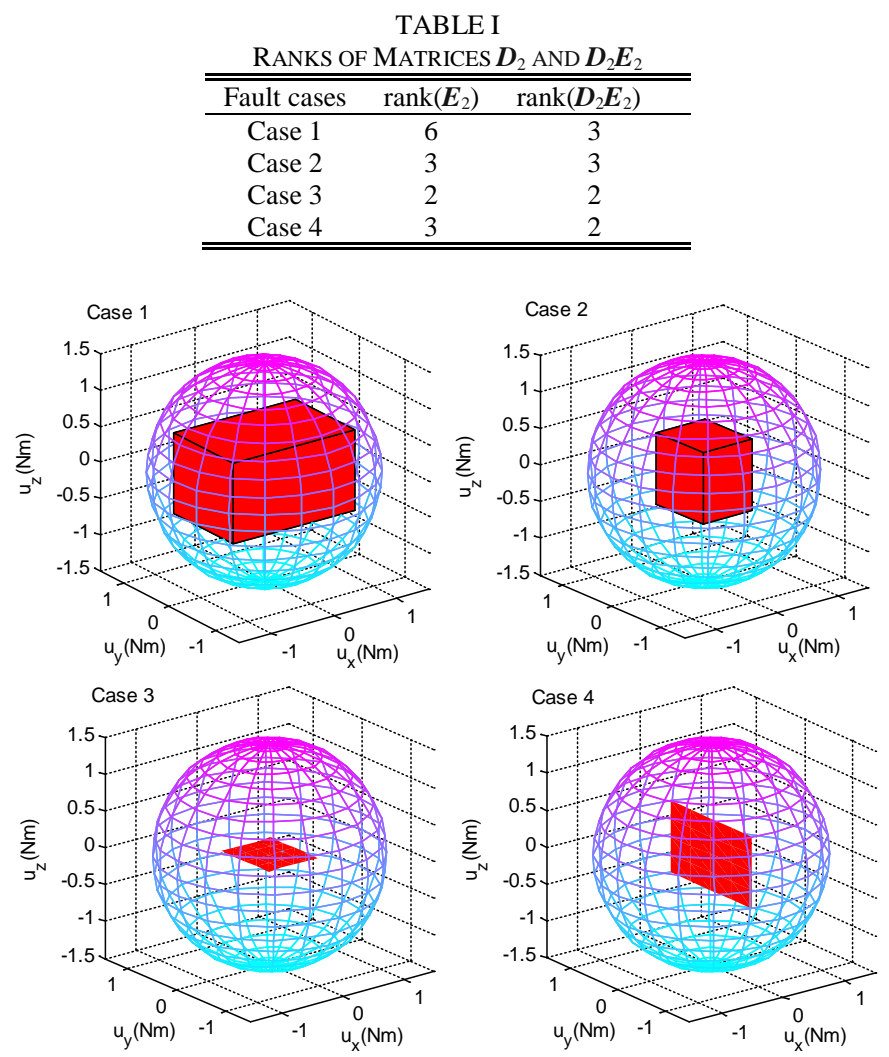

Fig. 4. Attainable sets of control torques under different fault cases (all sets are in a sphere whose radius is 1.5).

As can be seen the remaining active thrusters are able to produce a sufficient actuating power (to some extent at least) for the pursuer to perform some given maneuvers when the attitude control system suffers from the fault cases 1 and 2 . This is a straightforward result owing to $\operatorname{rank}\left(\boldsymbol{D}_{2} \boldsymbol{E}_{2}^{1}\right)=3$ and $\operatorname{rank}\left(\boldsymbol{D}_{2} \boldsymbol{E}_{2}^{2}\right)=3$ (see Table I). For the third case, $\operatorname{rank}\left(\boldsymbol{E}_{2}^{3}\right)=2<3$ is such that $\operatorname{rank}\left(\boldsymbol{D}_{2} \boldsymbol{E}_{2}^{3}\right)=2<3$, the attitude control subsystem then becomes under-actuated as discussed earlier. It is evident by inspecting the bottom left subplot of Fig. 4 that the attainable set of control torques is a plane paralleling to $x y$-plane, and thus the attitude motion in $z$ direction cannot be achieved. For case 4, it is interesting to note that $\operatorname{rank}\left(\boldsymbol{D}_{2} \boldsymbol{E}_{2}^{3}\right)=2<3$, although $\operatorname{rank}\left(\boldsymbol{E}_{2}^{4}\right)=3$ is such that $\operatorname{rank}\left(\boldsymbol{E}_{2}\right) \geq 3$ holds. Similar to case 3, this case also leads to an under-actuated system, and the attitude motion in $x$ direction is uncontrollable.

\section{SimULATION EXAMPLES}

In this section, numerical simulations performed on the 6-DOF coupled relative motion dynamics model with control law (52) and adaptive law (36) to validate the effectiveness of the proposed control scheme are implemented in the MATLAB/Simulink software environment. A freely tumbling satellite in an eccentric reference orbit with inertia matrix

$$
\boldsymbol{J}_{t}=\left[\begin{array}{ccc}
22 & 0.2 & 0.5 \\
0.2 & 20 & 0.3 \\
0.5 & 0.3 & 23
\end{array}\right]
$$

is referred to as the target spacecraft, and its orbit elements are shown in Table II. The target starts with initial attitude $\boldsymbol{q}_{t}=[0,0,0,1]^{T}$ and angularity velocity $\boldsymbol{\omega}_{i t}^{t}=[0.01,0,-0.01]^{T}$. The main control parameters as well as other simulation parameters are shown in Table III.

TABLE II

TARGET ORBIT ELEMENTS

\begin{tabular}{ccc}
\hline \hline Orbit elements & Value & Units \\
\hline$e$ & 0.1375 & -- \\
$i$ & 30 & $\mathrm{deg}$ \\
$\omega$ & 45 & $\mathrm{deg}$ \\
$\Omega$ & 50 & $\mathrm{deg}$ \\
$a$ & 6920 & $\mathrm{~km}$ \\
$v(0)$ & 15 & $\mathrm{deg}$ \\
\hline \hline
\end{tabular}

TABLE III

\begin{tabular}{cc}
\multicolumn{2}{c}{ OTHER SIMULATION PARAMETERS FOR THE PURSUER } \\
\hline \hline Parameters & Value \\
\hline$m_{p}$ & $200 \mathrm{~kg}$ \\
$\boldsymbol{J}_{p}$ & {$[55,0.3,0.5 ; 0.3,65,0.2 ; 0.5,0.2,58] \mathrm{kg} \cdot \mathrm{m}^{2}$} \\
$\boldsymbol{q}_{p}(0)$ & {$[-0.1,0.5,-0.2, \mathrm{sqrt}(0.7)]^{T}$} \\
$\boldsymbol{\omega}_{i p}^{p}(0)$ & {$[-0.02,0.01,0.02]^{T} \mathrm{rad} / \mathrm{s}$} \\
$\rho(0)$ & {$[100,-50,80]^{T} \mathrm{~m}$} \\
$\dot{\rho}(0)$ & 0.1 \\
$k$ & 10 \\
$k_{1}$ & 0.01 \\
$\varepsilon$ & 100 \\
$\eta$ & 2 \\
$\kappa$ & 1 \\
$\beta(0)$
\end{tabular}

The external disturbances simulated are of the forms: 


$$
\begin{gathered}
\boldsymbol{d}_{t}=10^{-5} \times\left([-1.025,6.248,-2.415]^{T} \sin \left(2 \pi \sqrt{\frac{\mu}{a^{3}}} t\right)-[10,-15,10]^{T}\right) \mathrm{N} \\
\boldsymbol{d}_{r}=10^{-4} \times\left[\begin{array}{l}
3 \cos \left(10 \omega_{d} t\right)+4 \sin \left(3 \omega_{d} t\right)-10 \\
-1.5 \sin \left(2 \omega_{d} t\right)+3 \cos \left(5 \omega_{d} t\right)+15 \\
3 \sin \left(10 \omega_{d} t\right)-8 \sin \left(4 \omega_{d} t\right)+10
\end{array}\right] \mathrm{N} \cdot \mathrm{m}
\end{gathered}
$$

where $\omega_{d}=0.1$ and $\mu=3.986 \times 10^{5} \mathrm{~km}^{3} / \mathrm{s}^{2}$.

The desired relative distance is $5 \mathrm{~m}$, so the virtual desired relative position vector is specified as $\boldsymbol{r}_{d}=[-5,0,0]^{T}$. Initially, the pursuer has a position error $\rho_{e}=[101.5794,-54.5634$, 81.2961] $]^{T}$ with respect to $\rho_{d}$, and a relative attitude of $\boldsymbol{q}_{e}=[-0.1,0.5,-0.2, \operatorname{sqrt}(0.7)]^{T}$ relative to the target. The orbit actuators and attitude actuators are thrusters and reaction wheels respectively, and the control force of each thruster and control torque of each reaction wheel are saturated at $1 \mathrm{~N}$ and $0.5 \mathrm{~N} \cdot \mathrm{m}$. The four pairs of thrusters are assumed to be distributed symmetrically on the pursuer, as illustrated in Fig. 5 , and each pair of thrusters can provide bidirectional force. The positions and installation directions of all thruster pairs are summarized in Table IV. Hence, the distribution matrix of orbit thrusters is

$$
\boldsymbol{D}_{1}=\frac{\sqrt{2}}{2}\left[\begin{array}{cccc}
-1 & -1 & 0 & 0 \\
-1 & 1 & 1 & -1 \\
0 & 0 & -1 & -1
\end{array}\right]
$$

TABLE IV

POSITIONS AND INSTALLATION DiRECTIONS OF THE THRUSTER PAIRS

\begin{tabular}{ccc}
\hline \hline Thruster pair & Direction & Position(m) \\
\hline T1 & {$[-\sqrt{2} / 2,-\sqrt{2} / 2,0]$} & {$[-0.4,-0.4,0],[0.4,0.4,0]$} \\
T2 & {$[-\sqrt{2} / 2, \sqrt{2} / 2,0]$} & {$[-0.4,0.4,0],[0.4,-0.4,0]$} \\
T3 & {$[0, \sqrt{2} / 2,-\sqrt{2} / 2]$} & {$[0,0.4,-0.4],[0,-0.4,0.4]$} \\
T4 & {$[0,-\sqrt{2} / 2,-\sqrt{2} / 2]$} & {$[0,-0.4,-0.4],[0,0.4,0.4]$} \\
\hline \hline
\end{tabular}
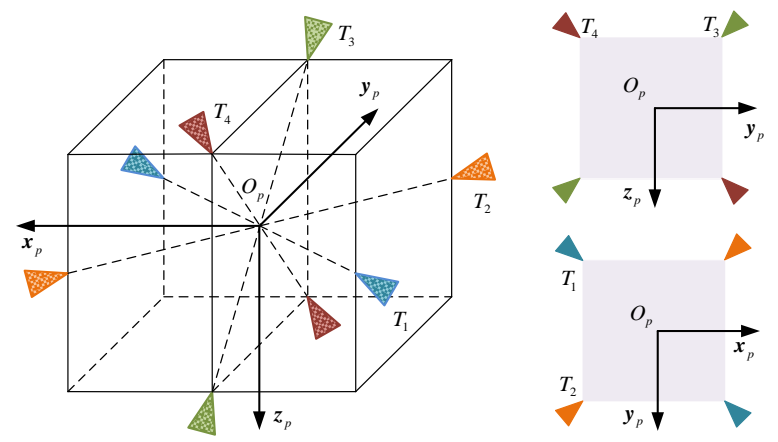

Fig. 5. The configuration structure of thrusters.

In addition, the attitude maneuvers are performed by four reaction wheels with classical configuration as shown in Fig. 6 (see Ref. [31]), and the corresponding distribution matrix is

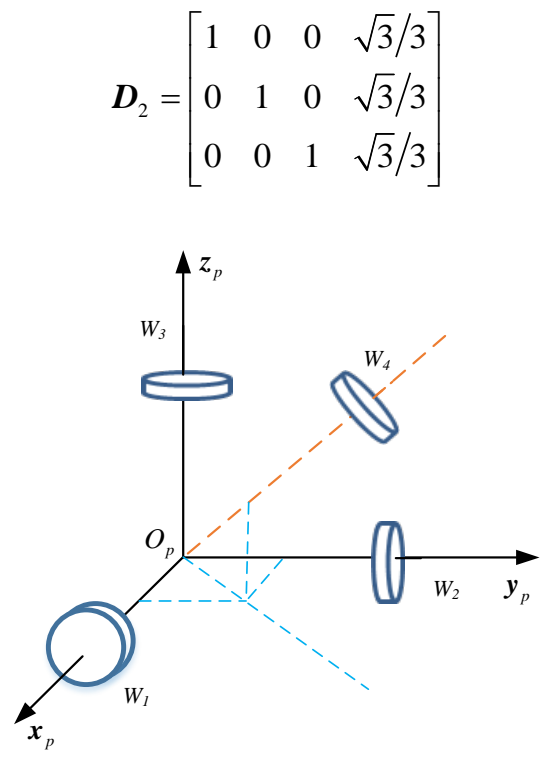

Fig. 6. The configuration of the flywheels.

\section{A. Simulation scenario without actuator faults}

In this scenario, all the actuators are assumed to work healthily. According to mission requirement, we set $t_{k}=50 \mathrm{~s}$, $t_{m}=375 \mathrm{~s}$ and $t_{f}=500 \mathrm{~s}$. Figure 7 shows that the relative orientation of the pursuer with respect to the target and the translational position error (expressed in the LVLH frame) between the relative position and fictitious desired relative position. The relative attitude has been transformed to attitude angles $\phi, \theta$ and $\psi$, which represent roll, pitch and yaw angles, respectively. As can be seen, the attitude tracking errors decay to a much smaller level of steady error less than $10^{-4} \mathrm{rad}$ within the prescribed terminal time $t_{f}=500 \mathrm{~s}$, and the pursuer is actuated by the thrusters to the desired position with steady accuracy better than $3 \times 10^{-3} \mathrm{~m}$. The time responses of the relative angular velocity and velocity error are depicted in Fig. 8. As is evident in Figs. 7 and 8, the proximity mission is achieved within 500s, and hereafter the translational and rotational motions of the two spacecraft keep synchronous.

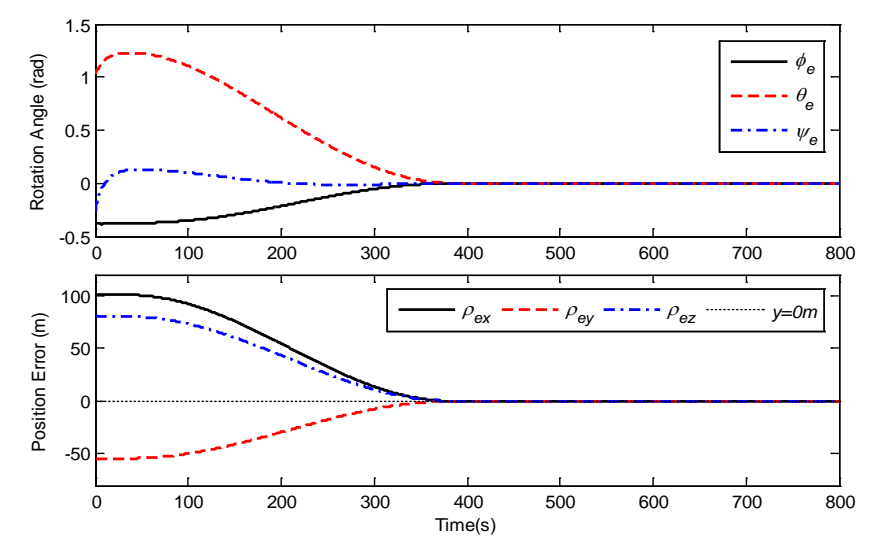

Fig. 7. Time responses of relative orientation and position error. 

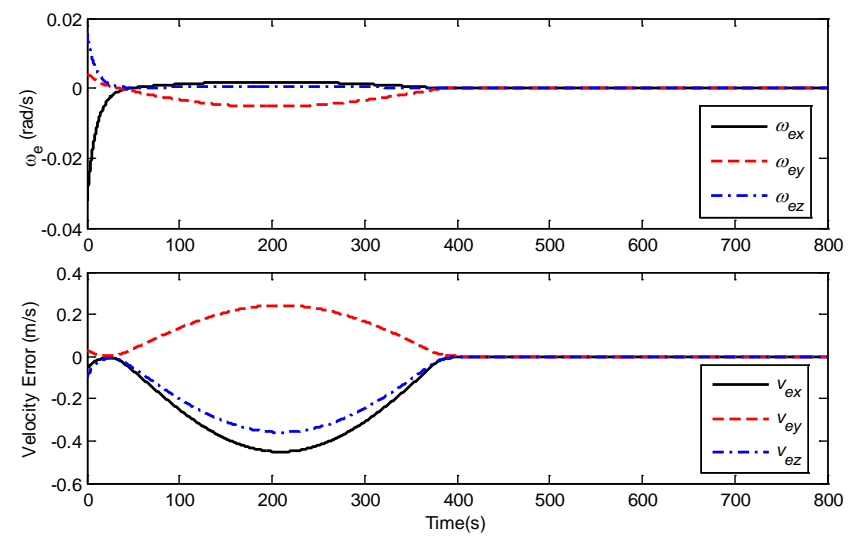

Fig. 8. Time responses of relation angular velocity and velocity error.

Moreover, polar curves are introduced to facilitate illustration of the trajectories of the relative position and attitude. Let $\|\boldsymbol{\rho}\|$ denote the relative distance of the pursuer relative to the target; $\alpha_{x}, \alpha_{y}$ and $\alpha_{z}$ denote the direction angles that relative position vector $\boldsymbol{\rho}$ makes with the $x-, y$ - and $z$-axes of the target's body frame, respectively; and $\Theta=2 \arccos \left(q_{e 4}\right)$ denote the rotation angle between the body-fixed frames of the pursuer and target. As such, the integrated position state (\|p\|, $\left.\alpha_{x}, \alpha_{y}, \alpha_{z}\right)$ in the polar coordinate describes the trajectory of the relative position vector $\boldsymbol{\rho}$, whereas $\|\boldsymbol{\rho}\|$ and $\Theta$ shows the tracking process of the relative translational and rotational motions, respectively. Figure 9 depicts the polar curves of the states $\left(\|\boldsymbol{\rho}\|, \alpha_{x}, \alpha_{y}, \alpha_{z}\right)$ and $(\|\boldsymbol{\rho}\|, \Theta)$. It can be seen that the relative position state $\left(\|\boldsymbol{\rho}\|, \alpha_{x}, \alpha_{y}, \alpha_{z}\right)$ ultimately converges to $\left(5,180^{\circ}, 0^{\circ}, 0^{\circ}\right)$, which means that the pursuer indeed reaches the desired position $\boldsymbol{r}_{d}=[-5,0,0]^{T}$ as expected. Moreover, from the convergence process of the state $(\|\boldsymbol{\rho}\|, \Theta)$, we know that the attitude tracking is achieved synchronously with the position maneuver, thus the control objective is achieved.

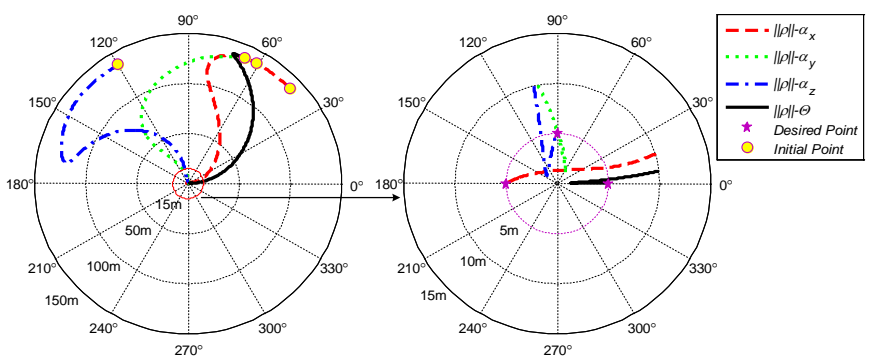

Fig. 9. Trajectories of relative position and orientation in polar coordinate.

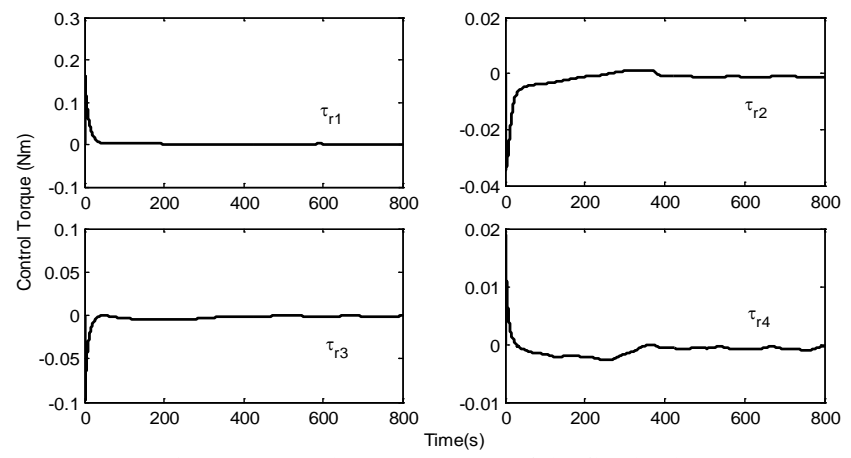

Fig. 10. Control torque outputs of the flywheels.

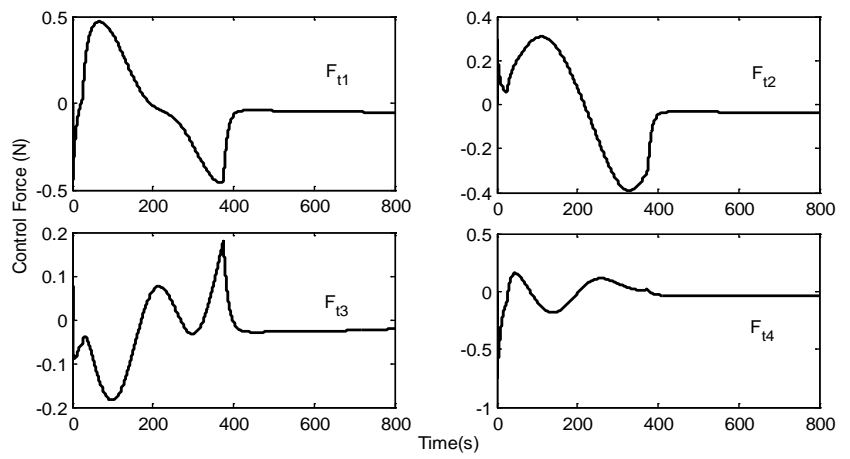

Fig. 11. Control force outputs of the thrusters.

The control histories of the rotational and translational motions are given in Figs. 10 and 11. It is shown that all the reaction wheels and thrusters work effectively to provide sufficient actuating torques and forces for the pursuer, thus guaranteeing the success of the capture mission. In addition, it is clear that all the actuators meet the magnitude limits.

Furthermore, to evaluate the impact of the different choices of $t_{m}$ on the required control effort pertaining to the initial period, a performance index function which stems from the optimal control problem is introduced and defined as

$$
E=\int_{0}^{t} \boldsymbol{u}_{c}^{T}(\tau) \boldsymbol{u}_{c}(\tau) d \tau
$$

where $t$ is the simulation time that is specified as 800s. Fig.12 shows the bar graphic visualizations of the control effort comparisons (here the actuator magnitude limits are not considered). It is obvious that reducing $t_{m}$ leads to a larger control effort at the initial period. Thus, the designer should trade off the control effort and the convergence rate through an appropriate choice of $t_{m}$; otherwise, the actuator outputs may exceed the magnitude limits, which may lead to a potential performance degradation and even instability.

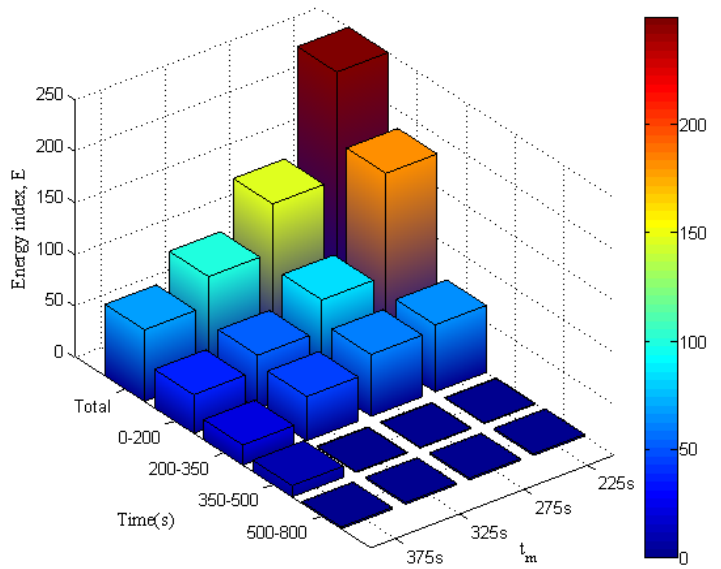

Fig. 12. Bar graphic visualizations of the control effort comparisons with different $t_{m}$.

\section{B. Simulation scenario with actuator faults}

In this scenario, the fault-tolerance ability of the proposed control scheme will be evaluated, and a severe case in which the thrusters and reaction wheels suffer from multiple faults is 
considered. The scenarios of the actuator faults simulated are outlined in Fig. 13, in which $\tau_{i}$ and $F_{i}$ respectively denote the commanded control signals of the reaction wheels and thrusters, $\tau_{r i}$ and $F_{t i}$ respectively denote the actual outputs of the reaction wheels and thrusters, and the health level of each actuator is specified by [21]

$$
\delta_{j}=0.7+0.15 \operatorname{rand}\left(t_{j}\right)+0.1 \sin (0.5 t+j \pi / 4), \quad j=1,2, \cdots, 8
$$

where $\operatorname{rand}(t)$ is a random number generator whose triggering condition is $t=0$, and the generated random number will be held until the next triggering condition occurs; $t_{j}=\bmod \left(t+\Delta t_{j}\right.$, $\Delta T$ ) with $\Delta t_{j}=0.4(j-1) \mathrm{s}$ and $\Delta T=3.2 \mathrm{~s}$ which denote the time delay and generation interval, respectively.

From Fig. 13, we know that $\boldsymbol{D} \boldsymbol{E} \boldsymbol{D}^{T}$ remains positive definite all the time, which implies that the proposed controller can deal with this fault case effectively. With the same parameters as the previous simulation scenario, the time responses of the system states are shown in Fig. 14. The relative angular velocity and velocity errors are shown in Fig. 15. It can be seen that the tracking mission can be achieved within the prescribed time $t_{f}$, with steady errors of relative attitude and position less than $1.5 \times 10^{-4} \mathrm{rad}$ and $3 \times 10^{-3} \mathrm{~m}$.

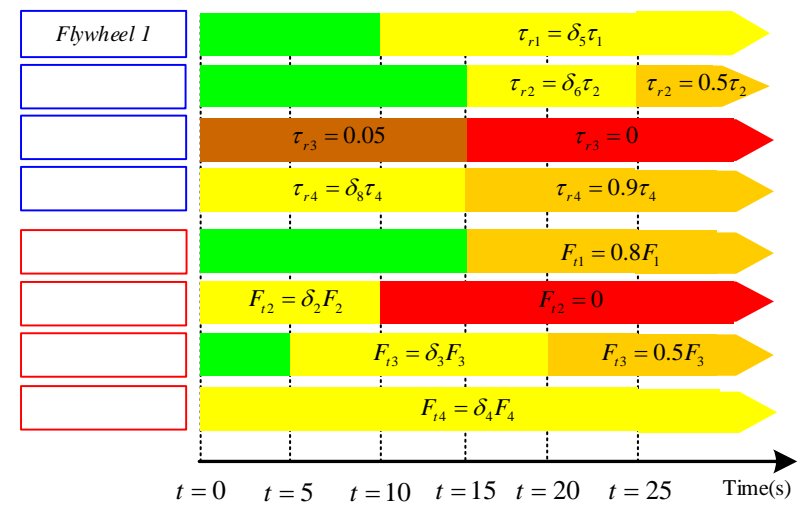

Fig. 13. Fault scenarios of flywheels and thrusters.
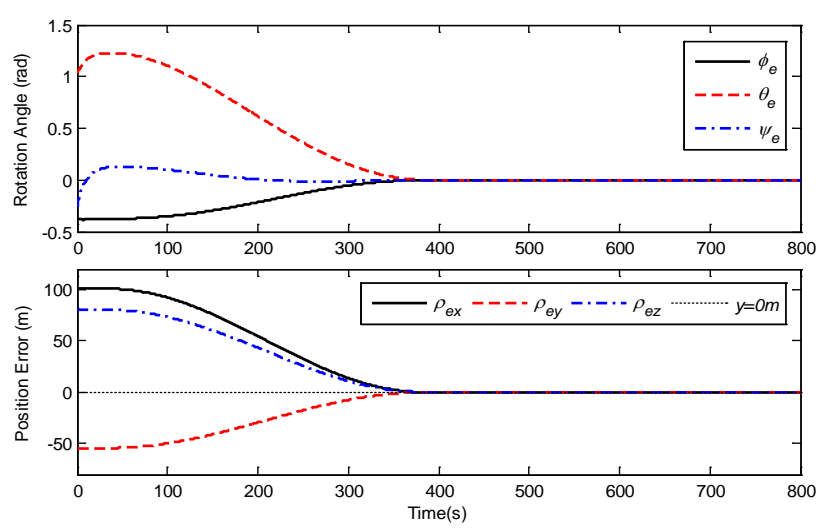

Fig. 14. Time responses of system states for the faulty case.
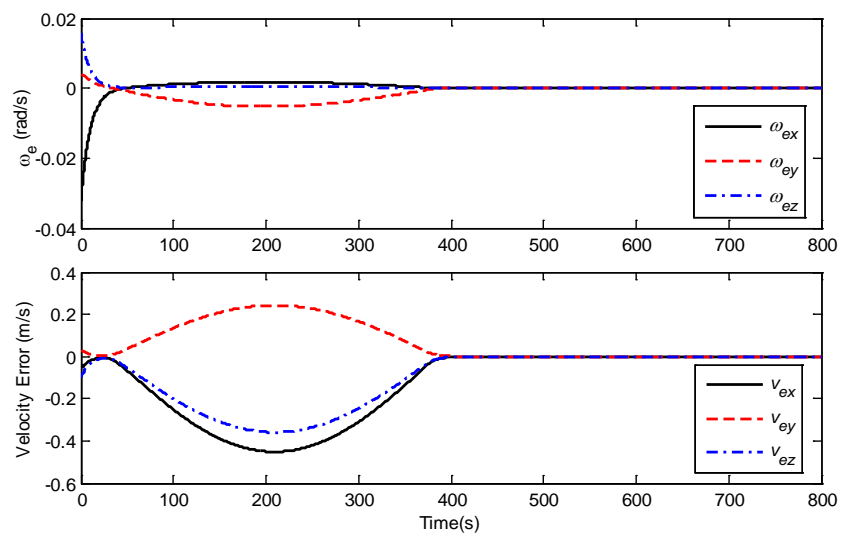

Fig. 15. Time responses of relative angular velocity and velocity error.

To demonstrate the approaching process, the 3-D trajectory of the relative position expressed in the target's body-fixed frame is shown in Fig. 16. It clearly illustrates that the relative position vector converges to the desired position $\boldsymbol{r}_{d}=[-5,0,0]^{T}$, which indicates that the docking port of the target indeed points towards the mass center of the pursuer. Furthermore, to illustrate the attitude tracking motion, a series of snapshots of the actual attitude orientation of the pursuer (see from the target reference frame) are given in Fig. 17. It is intuitively shown that the attitude synchronization is achieved within 500s, and hereafter the docking port of pursuer is aligned with the receiving component of the target. As a consequence, the control objective is achieved.

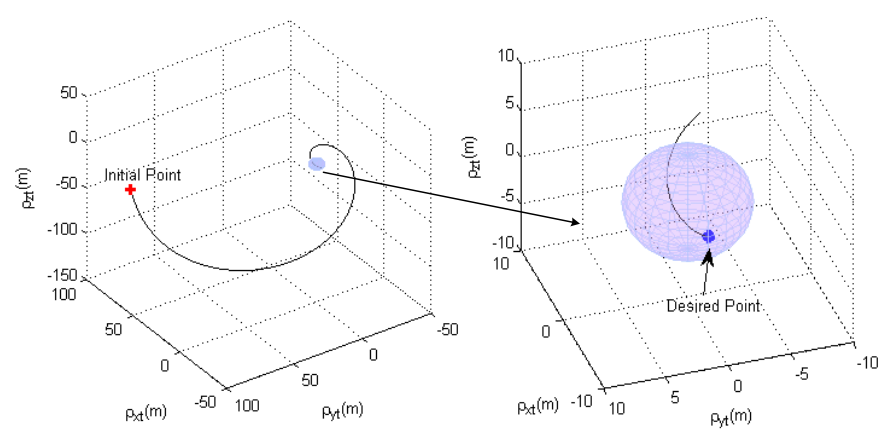

Fig. 16. Trajectory of the relative position in the target body-fixed frame.

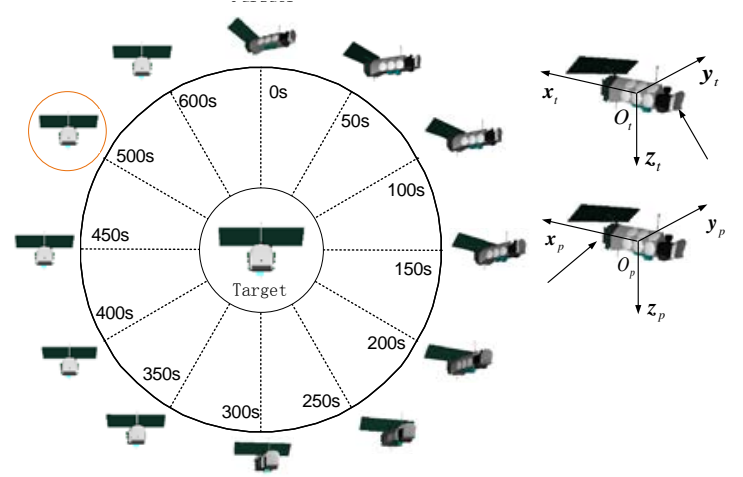

Fig. 17. Snapshots of the attitude orientation history.

Figures 18 and 19 show the actual outputs of the reaction wheels and thrusters, respectively. As can be seen, the remaining active reaction wheels and thrusters are still able to 
guarantee the success of the proximity mission, although some actuators suffer from server faults. Additionally, the burrs appearing in the actual outputs of the actuators are mainly caused by the random actuator faults.

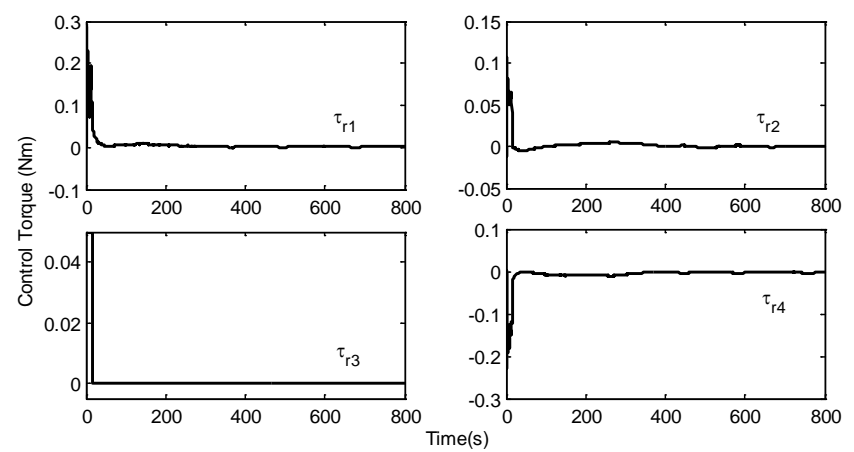

Fig. 18. Control torque outputs of the flywheels for the faulty case.

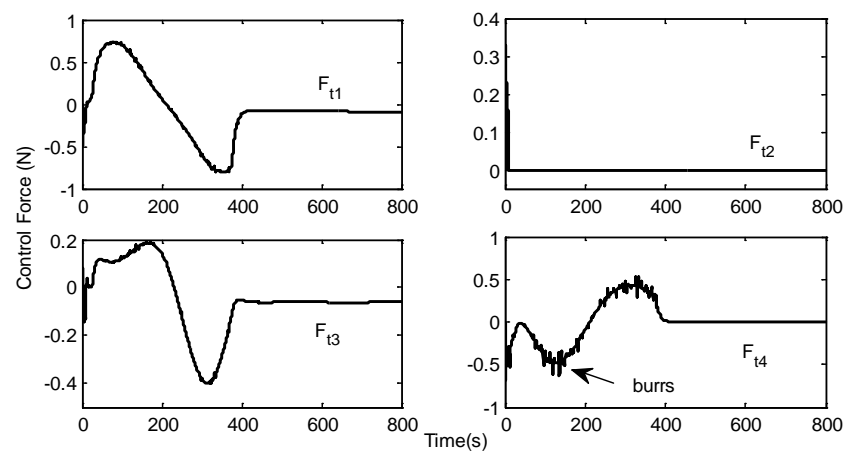

Fig. 19. Control force outputs of the thrusters for the faulty case.

To further demonstrate the fault-tolerance ability, we compare the proposed control scheme (noted as FFTC) with the adaptive backstepping control (noted as ABSC) developed in [17] and the widely used proportional-derivative control (noted as PD) in [32]. For fair comparison, the control parameters of the ABSC and PD are determined by trial and error such that these three control schemes have nearly identical convergence rates for the healthy case. For the healthy case, the detailed control performance comparisons in terms of steady-state accuracy are provided in Table V, and the 3-D trajectories of relative attitude and position error are given in Figs. 20 and 21. It is shown that all three controllers can finally accomplish the close-proximity mission. For the faulty case, the 3-D trajectories of relative attitude and position error are shown in Figs. 22 and 23, respectively. Together with the performance comparisons as in Table V, it is apparent that the ABSC and the PD controllers have a limited fault-tolerance ability to the given fault case, and cannot guarantee the safe and effective proximity operations due to the poor performance in terms of tracking accuracy. In contrast, the proposed control scheme can still obtain high control precision and good tracking performance even in the event of the actuator faults as described above.

TABLE IV

CONTROL PERFORMANCE COMPARISONS

\begin{tabular}{cccc}
\hline & & $\begin{array}{l}\text { Steady error of } \\
\boldsymbol{\rho}_{e}(\mathrm{~m})\end{array}$ & $\begin{array}{l}\text { Steady error of } \\
\text { rotation angle (rad) }\end{array}$ \\
\hline Healthy & FFTC & $3 \times 10^{-3}$ & $7 \times 10^{-5}$ \\
Case & ABSC & $1 \times 10^{-1}$ & $8 \times 10^{-3}$ \\
& PD & $1.5 \times 10^{-1}$ & $1.5 \times 10^{-2}$ \\
& FFTC & $3 \times 10^{-3}$ & $1.5 \times 10^{-4}$ \\
Faulty & ABSC & -- & $2.5 \times 10^{-3}$ \\
Case & PD & $7 \times 10^{-1}$ & $1.6 \times 10^{-1}$ \\
\hline \hline
\end{tabular}

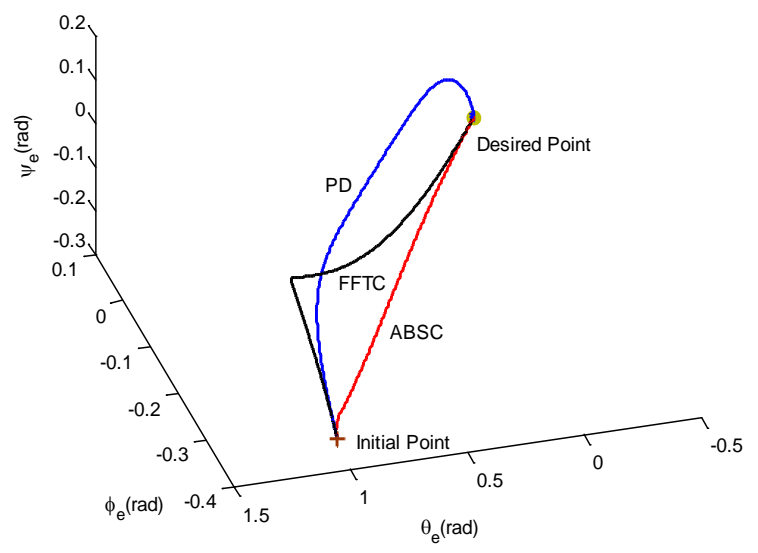

Fig. 20. Trajectories of relative orientation for the healthy case.

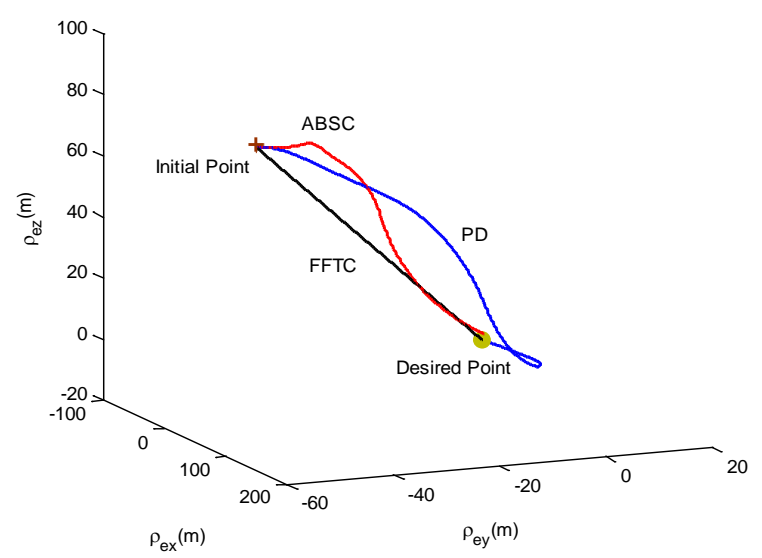

Fig. 21. Trajectories of position error for the healthy case.

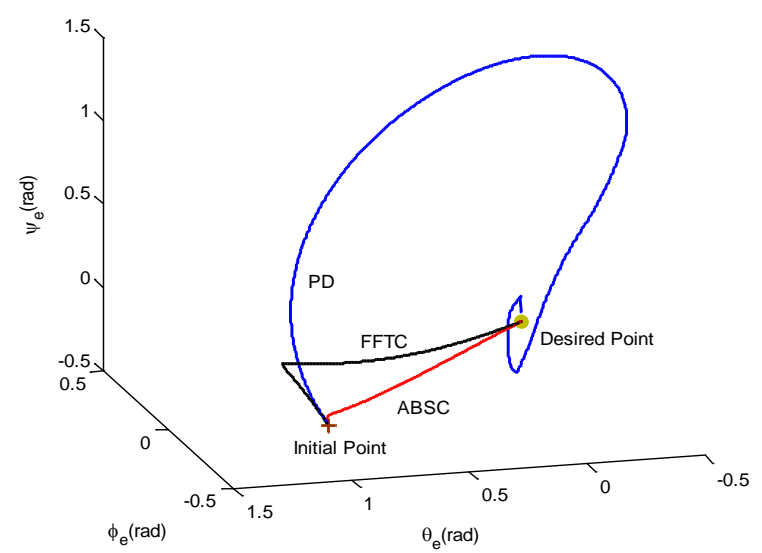

Fig. 22. Trajectories of relative orientation for the faulty case. 


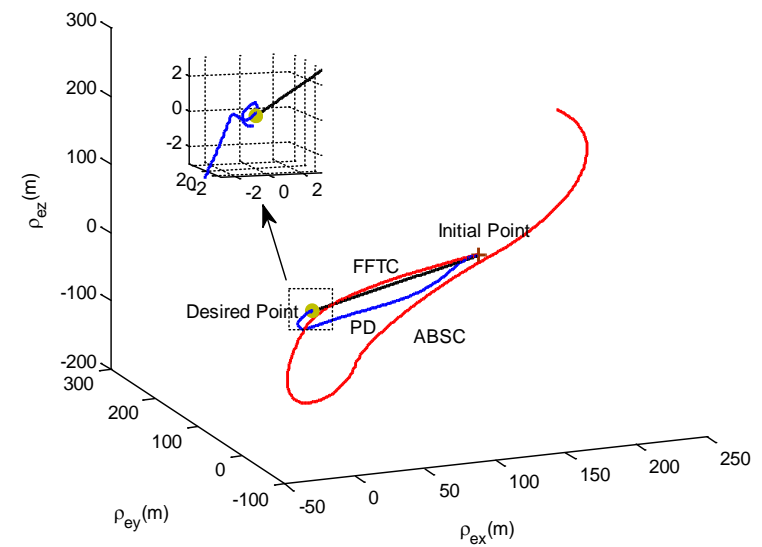

Fig. 23. Trajectories of position error for the faulty case.

Summarizing all the simulation scenarios, the proposed controller shows superiority in control performance for the spacecraft proximity operations, despite the presence of actuator faults, external disturbances, and uncertain mass and inertia properties of the pursuer. In addition, an outstanding feature residing in the proposed control scheme is that the tracking mission can be accomplished in a prescribed time. Moreover, the flexibility in the parameter selection can be utilized to obtain the desired performance.

\section{CONCLUSION}

In this paper, a novel solution to the tracking control problem of proximity operations between a maneuverable pursuer and a tumbling free-flying object in orbit has been presented. A robust fault-tolerant control strategy was developed that seeks to align the relative position vector along the docking port of the target while maintaining a safe relative distance, and synchronously to reorient the pursuer such that its docking port is always facing the docking component of the target. The control scheme was shown to achieve accurate relative position tracking as well as attitude synchronization, despite the presence of external disturbances, parametric uncertainties and actuator faults. Moreover, the translational and rotational tracking errors were guaranteed to converge to zero within a prescribed time. Furthermore, a rigorous theoretical analysis for the fault-tolerance ability of the designed controller was also presented, which shows that the control scheme can ensure the success of proximity operations for a large class of actuator faults. Numerical simulations have been presented to illustrate the performance of the developed control algorithm in terms of disturbance suppression, finite-time convergence and faulttolerance ability. However, how to guarantee collision avoidance in the event of those kinds of possible actuator faults that beyond the controller's fault-tolerance ability (a design aspect of practical significance in decreasing the likelihood of huge economic losses) is not considered in this paper. This is thought to be a field where further study is called for.

\section{APPENDIX}

PROOF OF LEMMA 3

Suppose that $\operatorname{rank}(\boldsymbol{D E})=6$ remains valid all the time for the given fault cases. Let $\boldsymbol{E}=\boldsymbol{E}^{1 / 2} \boldsymbol{E}^{1 / 2}$, which is a reasonable manipulation due to each element of $\boldsymbol{E}_{i}$ satisfies $0 \leq \delta_{i j} \leq 1$. According to $\operatorname{rank}(\boldsymbol{D E}) \leq \min \left\{\operatorname{rank}\left(\boldsymbol{D} \boldsymbol{E}^{1 / 2}\right), \operatorname{rank}\left(\boldsymbol{E}^{1 / 2}\right)\right\}$, it is then straightforward to obtain that

$$
\operatorname{rank}\left(\boldsymbol{D} \boldsymbol{E}^{1 / 2}\right)=6
$$

Notice that, by using the preceding manipulation, the matrix $\boldsymbol{D E} \boldsymbol{D}^{T}$ can be transformed to

$$
\boldsymbol{D} \boldsymbol{E} \boldsymbol{D}^{T}=\boldsymbol{D} \boldsymbol{E}^{1 / 2} \boldsymbol{E}^{1 / 2} \boldsymbol{D}^{T}=\boldsymbol{D} \boldsymbol{E}^{1 / 2}\left(\boldsymbol{D} \boldsymbol{E}^{1 / 2}\right)^{T}=\boldsymbol{A} \boldsymbol{A}^{T}
$$

where $\boldsymbol{A} \in \square^{6 \times n}$ is used here to denote $\boldsymbol{D} \boldsymbol{E}^{1 / 2}$.

We now know directly from Eq. (A1) that $A^{T}$ is a full column rank matrix, that is, $\operatorname{rank}\left(\boldsymbol{A}^{T}\right)=6$. Then, consider the following homogeneous linear equations

$$
\boldsymbol{A}^{T} \boldsymbol{X}=\mathbf{0}
$$

It is apparent that the homogeneous system in Eq. (A3) possesses a unique solution, that is, the trivial solution $\boldsymbol{x}=\mathbf{0}$, since $\operatorname{rank}\left(\boldsymbol{A}^{T}\right)=6$ is such that there are no free variables (See Ref. [33]). In other words, for any $\boldsymbol{x} \in \square^{6 \times 1} \neq \mathbf{0}, \boldsymbol{A}^{T} \boldsymbol{x} \neq \mathbf{0}$ always holds true.

As indicated above, given any vector $\boldsymbol{y} \in \square^{6 \times 1} \neq \mathbf{0}$, it follows that

$$
\boldsymbol{y}^{T} \boldsymbol{D E} \boldsymbol{D}^{T} \boldsymbol{y}=\boldsymbol{y}^{T} \boldsymbol{A} \boldsymbol{A}^{T} \boldsymbol{y}=\left(\boldsymbol{A}^{T} \boldsymbol{y}\right)^{T} \boldsymbol{A}^{T} \boldsymbol{y}>0
$$

Thus, we conclude that the matrix $\boldsymbol{D E} \boldsymbol{D}^{T}$ is positive definite.

Now suppose that all the fault cases are such that $\boldsymbol{D E} \boldsymbol{D}^{T}$ remains positive definite, it is a straightforward matter to show that $\operatorname{rank}\left(\boldsymbol{D E} \boldsymbol{D}^{T}\right)=6$. Then, by making use of the relationship $\operatorname{rank}\left(\boldsymbol{D E} \boldsymbol{D}^{T}\right) \leq \min \left\{\operatorname{rank}(\boldsymbol{D E}), \operatorname{rank}\left(\boldsymbol{D}^{T}\right)\right\}$, and noting that $\boldsymbol{D}$ is a full-row rank matrix, that is, $\operatorname{rank}(\boldsymbol{D})=6$, we present the necessary condition to obtain the condition $\operatorname{rank}\left(\boldsymbol{D E} \boldsymbol{D}^{T}\right)=6$ is that

$$
\operatorname{rank}(\boldsymbol{D E}) \geq 6
$$

With regard to Eq. (A5), it is evident that $\operatorname{rank}(\boldsymbol{D E})=6$ due to the fact that $\boldsymbol{D E} \in \square^{6 \times n}$ with $n>6$. This completes the proof.

\section{REFERENCES}

[1] K. Subbarao and S. J. Welsh, "Nonlinear control of motion synchronization for satellite proximity operations," Journal of Guidance, Control, and Dynamics, vol. 31, no. 5, pp. 1284-1294, Sep./Oct. 2008.

[2] F. Aghili, "Automated rendezvous \& docking (AR\&D) without impact using a reliable 3D vision system," in Proceedings of the AIAA Guidance, 
Navigation, and Control Conference, Toronto, Ontario Canada, Aug. 2-5, 2010, pp. 7602.

[3] J. Ventura, M. Ciarcià, M. Romano, and U. Walter, "An inverse dynamics-based trajectory planner for autonomous docking to a tumbling target," in Proceedings of the AIAA Guidance, Navigation, and Control Conference, San Diego, California, USA, Jan. 4-8, 2016, pp. 0876.

[4] I. Kawano, M. Mokuno, T. Kasai, and T. Suzuki, "Result of autonomous rendezvous docking experiment of engineering test satellite-VII," Journal of Spacecraft and Rockets, vol. 38, no. 1, pp. 105-111, Jan./Feb. 2001.

[5] D. C. Woffinden and D. K. Geller, "Navigating the road to autonomous orbital rendezvous," Journal of Spacecraft and Rockets, vol. 44, no. 4, pp. 898-909, Jul./Aug. 2007.

[6] W. H. Clohessy, "Terminal guidance system for satellite rendezvous," Journal of the Aerospace Sciences, vol. 27, no. 9, pp. 653-658, 1960.

[7] T. Carter and M. Humi, "Fuel-optimal rendezvous near a point in general Keplerian orbit,” Journal of Guidance, Control, and Dynamics, vol. 10, no. 6, pp. 567-573, Nov./Dec.1987.

[8] G. Inalhan, M. Tillerson, and J. P. How, "Relative dynamics and control of spacecraft formations in eccentric orbits," Journal of Guidance, Control, and Dynamics, vol. 25, no.1, pp. 48-59, Jan./Feb. 2002.

[9] R. G. Melton, "Time-explicit representation of relative motion between elliptical orbits," Journal of Guidance, Control, and Dynamics, vol. 23, no. 4, pp. 604-610, Jul./Aug. 2000.

[10] R. Kristiansen and P. J. Nicklasson, "Spacecraft formation flying: a review and new results on state feedback control," Acta Astronautica, vol. 65, no. 11, pp. 1537-1552, Dec. 2009.

[11] H. Yoon and B. N. Agrawal, "Novel expressions of equations of relative motion and control in Keplerian orbits," Journal of Guidance, Control, and Dynamics, vol. 32, no. 2, pp. 664-669, Mar./Apr. 2009.

[12] S. Segal and P. Gurfil, "Effect of kinematic rotation-translation coupling on relative spacecraft translational dynamics," Journal of Guidance, Control, and Dynamics, vol. 32, no. 3, pp. 1045-1050, May/Jun. 2009.

[13] D. Lee and G. Vukovich, "Kinematically coupled spacecraft relative motion without attitude synchronization assumption," Aerospace Science and Technology, vol. 45, pp. 316-323, Sep. 2015.

[14] H. Pan and V. Kapila, "Adaptive nonlinear control for spacecraft formation flying with coupled translational and attitude dynamics," in Proceedings of the 40th IEEE Conference on Decision and Control, Orlando, FL, USA, Dec. 4-7, 2001, pp. 2057-2062.

[15] W. Lu, Y. H. Geng, X. Q. Chen, and F. Zhang, "Relative position and attitude coupled control for autonomous docking with a tumbling target," International Journal of Control and Automation, vol. 4, no. 4, pp. 1-22, Dec. 2011.

[16] N. Filipe and P. Tsiotras, "Adaptive position and attitude-tracking controller for satellite proximity operations using dual quaternions," Journal of Guidance, Control, and Dynamics, vol. 38, no. 4, pp. 566-577, Apr. 2014.

[17] L. Sun and W. Huo, "6-DOF integrated adaptive backstepping control for spacecraft proximity operations," IEEE Transactions on Aerospace and Electronic Systems, vol. 51, no. 3, pp. 2433-2443, Jul. 2015.

[18] F. Zhang and G. R. Duan, "Robust adaptive integrated translation and rotation finite-time control of a rigid spacecraft with actuator misalignment and unknown mass property,” International Journal of Systems Science, vol. 45, no. 5, pp. 1007-1034, 2014.

[19] L. Sun, "Passivity-based adaptive finite-time trajectory tracking control for spacecraft proximity operations," Journal of Spacecraft and Rockets, vol. 53, no. 1, pp. 46-56, Jan./Feb. 2016.

[20] S. Croomes, "Overview of the DART mishap investigation results," NASA Report, pp. 1-10, $2006 . \quad$ [Online]. Available: https://www.nasa.gov/pdf/148072main_DART_mishap_overview.pdf

[21] W. Cai, X. Liao, and D. Y. Song, "Indirect robust adaptive fault-tolerant control for attitude tracking of spacecraft," Journal of Guidance Control and Dynamics, vol. 31, no. 5, pp. 1456-1463, Sep./Oct. 2008.

[22] B. Xiao, Q. Hu, and Y. Zhang, "Finite-time attitude tracking of spacecraft with fault-tolerant capability," IEEE Transactions on Control Systems Technology, vol. 23, no. 4, pp. 1338-1350, Jul. 2015.

[23] L. S. Breger and J. P. How, "Safe trajectories for autonomous rendezvous of spacecraft," Journal of Guidance, Control, and Dynamics, vol. 31, no. 5, pp. 1478-1489, Sep./Oct. 2008.

[24] S. Jayaram, "Fault tolerant autonomous rendezvous and docking architecture for spacecraft in presence of control actuator failures," International Journal of Intelligent Unmanned Systems, vol. 1, no. 1, pp. 5-20, 2013.
[25] H. Dong, Q. Hu, and G. Ma, "Dual-quaternion based fault-tolerant control for spacecraft formation flying with finite-time convergence," ISA transactions, vol. 61, pp. 87-94, Mar. 2016.

[26] R. J. Wallsgrove and M. R. Akella, "Globally stabilizing saturated attitude control in the presence of bounded unknown disturbances," Journal of Guidance Control and Dynamics, vol. 28, no. 5, pp. 957-963, Sep./Oct. 2005.

[27] U. Lee and M. Mesbahi, "Feedback control for spacecraft reorientation under attitude constraints via convex potentials," IEEE Transactions on Aerospace and Electronic Systems, vol. 50, no. 4, pp. 2578-2592, Oct. 2014.

[28] B. T. Costic, D. M. Dawson, M. S. De Queiroz, and V. Kapila, "Quaternion-based adaptive attitude tracking controller without velocity measurements," Journal of Guidance, Control, and Dynamics, vol. 24, no. 6, pp. 1214-1222, Nov./Dec. 2001.

[29] Y. S. Lu, C. W. Chiu, and J. S. Chen, "Time-varying sliding-mode control for finite-time convergence," Electrical Engineering, vol. 92, pp. 257-268, Dec. 2010.

[30] R. A. DeCarlo, S. H. Zak, and G. P. Matthews, "Variable structure control of nonlinear multivariable systems: a tutorial," Proceedings of the IEEE, vol. 76, no. 3, pp. 212-232, Mar. 1988.

[31] Q. Hu, B. Li, and Y. Zhang, "Nonlinear proportional-derivative control incorporating closed-loop control allocation for spacecraft," Journal of Guidance Control and Dynamics, vol. 37, no. 3, pp. 799-812, May/Jun. 2014.

[32] R. Kristiansen, P. J. Nicklasson, and J. T. Gravdahl, "Spacecraft coordination control in 6DOF: Integrator backstepping vs passivity-based control," Automatica, vol. 44, no. 11, pp. 2896-2901, Nov. 2008.

[33] C. D. Meyer, Matrix Analysis and Applied Linear Algebra. Philadelphia, PA: SIAM, 2000.

[34] Z. Zhu, Y. Q. Xia, and M. Y. Fu, "Attitude stabilization of rigid spacecraft with finite-time convergence," International Journal of Robust and Nonlinear Control, vol. 21, no. 6, pp. 686-702, 2015. 